\title{
Do emotion-induced blindness and the attentional blink share underlying mechanisms? An event-related potential study of emotionally-arousing words
}

\author{
Jeffrey MacLeod ${ }^{1} \cdot$ Brandie M. Stewart $^{1} \cdot$ Aaron J. Newman $^{1} \cdot$ Karen M. Arnell $^{2}$
}

Published online: 6 March 2017

(C) Psychonomic Society, Inc. 2017

\begin{abstract}
When two targets are presented within approximately $500 \mathrm{~ms}$ of each other in the context of rapid serial visual presentation (RSVP), participants' ability to report the second target is reduced compared to when the targets are presented further apart in time. This phenomenon is known as the attentional blink (AB). The $\mathrm{AB}$ is increased in magnitude when the first target is emotionally arousing. Emotionally arousing stimuli can also capture attention and create an AB-like effect even when these stimuli are presented as to-be-ignored distractor items in a single-target RSVP task. This phenomenon is known as emotion-induced blindness (EIB). The phenomenological similarity in the behavioral results associated with the $\mathrm{AB}$ with an emotional $\mathrm{T} 1$ and EIB suggest that these effects may result from similar underlying mechanisms - a hypothesis that we tested using event-related electrical brain potentials (ERPs). Behavioral results replicated those reported previously, demonstrating an enhanced $\mathrm{AB}$ following an emotionally arousing target and a clear EIB effect. In both paradigms highly arousing taboo/sexual words resulted in an increased early posterior negativity (EPN) component that has been suggested to represent early semantic activation and selection for further processing in working memory. In both paradigms taboo/sexual words also produced an increased late positive potential (LPP) component that has been suggested to represent consolidation of a stimulus in working memory. Therefore, ERP results provide evidence that the EIB and emotionenhanced $\mathrm{AB}$ effects share a common underlying mechanism.
\end{abstract}

Aaron J. Newman

Aaron.Newman@dal.ca

1 Department of Psychology and Neuroscience, Life Sciences Centre, Dalhousie University, Box 15000, Halifax, NS B3H 4R2, Canada

2 Department of Psychology, Brock University, 500 Glenridge Avenue, St. Catharines, ON L2S 3A1, Canada
Keywords Language $\cdot$ Attention · Reading $\cdot$ Emotion · Event-related potentials $\cdot$ Attentional blink $\cdot$ Emotion-induced blindness $\cdot$ Rapid serial visual presentation

\section{Introduction}

Humans receive a variety of sensory inputs at any one time, and typically do not have the resources to process all inputs with equally. Attention is used to select the most important inputs for further processing and awareness. If attentional selection exists to prioritize the processing of important sensory inputs, stimuli that are relevant to the welfare of an individual, such as threat or sexual stimuli, should be efficiently selected for further processing by the attentional system. Indeed, human behavioral research using a variety of cognitive paradigms and participant groups has shown that emotionally charged stimuli are processed preferentially by the attentional system (e.g., Aquino \& Arnell, 2007; Frischen, Eastwood, \& Smilek, 2008; MacKay, Shafto, Taylor, Marian, Abrams, \& Dyer, 2004; Vogt, De Houwer, Koster, Van Damme, \& Crombez, 2008). An examination of attention to emotional words in rapid serial visual presentation (RSVP) is the focus of the current work.

In RSVP, stimuli are presented one-at-a-time at a central location with approximately $100 \mathrm{msec}$ separating each item. This method of presentation causes each item to mask the previously presented item. Participants are typically able to identify a single target within an RSVP stream with a high level of accuracy. However, if participants are asked to identify two targets within a single RSVP stream, performance on the second target (T2) is detected with reduced accuracy when it appears less than $500 \mathrm{~ms}$ after the first target (T1; Raymond, Shapiro, \& Arnell, 1992). This phenomenon is called the attentional blink (AB; Raymond et al., 1992). 
Emotionally arousing stimuli have consistently been found to modulate the AB. When emotionally arousing words are presented in the $\mathrm{T} 1$ position, they result in a larger and more prolonged AB compared to non-arousing T1 words (Mathewson, Arnell, \& Mansfield, 2008). Emotionally arousing words presented as T2 are less susceptible to the AB than non-arousing T2 words (Anderson, 2005; Keil \& Ihssen, 2004; Milders et al., 2006). However, emotional T2 words are more susceptible to the AB when preceded by an emotional T1 word (Schwabe \& Wolf, 2010; Schwabe et al., 2011). Together these results suggest that emotional stimuli receive additional attentional resources at the expense of neutral stimuli in the AB paradigm.

Emotionally arousing stimuli have also been presented as to-be-ignored distractor items in RSVP tasks (e.g., Most, Chun, Widders, \& Zald, 2005; Arnell, Killman, \& Fijavz, 2007). In these tasks, the emotional distractor appears prior to the target item, just as T1 would precede T2 in a typical AB task - but unlike in the AB task, these distractor items are not relevant to any task the participant is instructed to perform (see Fig. 1b for a depiction). In this emotional-distractor paradigm, emotionally arousing distractors have been shown to capture attention at the expense of accuracy for closely trailing target items; similar to T1 capturing attention at the expense of T2 in the AB paradigm (Arnell et al., 2007; Mathewson et al., 2008; Most et al., 2005; Most, Smith, Cooter, Levy, \& Zald, 2007). This effect has been termed emotion-induced blindness (EIB; Most et al., 2005) or the emotional attentional blink (McHugo, Olatunji, \& Zald, 2013). For example, Arnell and colleagues (2007) showed that relative to emotionally-neutral words, a sexual/taboo distractor word (e.g., orgasm, bitch) captured attention and resulted in an EIB for neutral word targets presented soon after the emotional word. Higher arousal ratings and greater surprise recognition memory for the emotional distractor word were found to predict the accuracy for subsequent targets presented at short lags, and memory fully mediated the relationship between arousal rating and target accuracy. Arnell et al. (2007) concluded that arousing distractor words were encoded into memory at the expense of targets presented at short lags following the distractor.

\section{The AB and EIB: Similar mechanisms?}

Mathewson et al. (2008) showed that similar patterns of behavioral data result from $\mathrm{AB}$ tasks with emotionally arousing T1 words, and EIB tasks with emotionally arousing distractors. Both with the AB and EIB, higher arousal ratings and greater surprise recognition memory for the emotional words were found to predict reduced accuracy for subsequent targets presented at short lags, and memory fully mediated the relationship between arousal rating and target accuracy. Mathewson et al. (2008) used the same words in the two tasks and used a correlational analysis to show that the words that reduced T2 accuracy when presented as T1 in an AB task were highly similar to the words that reduced target accuracy when presented as to-be-ignored distractor words in the EIB task. As discussed by Mathewson and colleagues (2008), these results suggest that the same mechanism may underlie the effect of emotionally arousing words in both tasks.

An important distinction between the standard emotional $\mathrm{AB}$ and EIB is the task-relevance of the emotional word. In both tasks, the emotional nature of the word is task-irrelevant. However, the word itself is task-relevant in the emotional AB and task-irrelevant in the EIB task. Although the similarities in the behavioral results presented above suggest that the same mechanisms may underlie the emotional $\mathrm{AB}$ and EIB, further investigation of this issue is warranted given the differences between these two tasks. The goal of the current experiments was to compare the $\mathrm{AB}$ and $\mathrm{EIB}$ effects using event-related brain potentials (ERPs) to determine whether common neural patterns underlie these two effects.

\section{Electrophysiological investigations of the AB}

The Central Interference Theory proposes two processing stages for detecting and remembering stimuli presented in RSVP (e.g., Chun \& Potter, 1995; Jolicoeur, 1999; Jolicoeur \& Dell'Acqua, 1998). According to this theory, there is an initial high-capacity processing stage in which perceptual and semantic representations of an object are formed. In this initial stage, features of stimuli, including conceptual representations relevant to target detection are processed. This initial stage is followed by a limited capacity serial stage in which selected stimuli receive sustained attention and are consolidated into working memory (Potter, 1993; Potter \& Lombardi, 1990). The AB has been proposed to occur when T2 is presented while T1 is still being consolidated into working memory. As such, T2 receives stage 1 processing but not stage 2 consolidation into WM.

Electrophysiological investigations of brain activity during $A B$ tasks have provided support for these two stages in the AB. Examinations of ERP components following the presentation of $\mathrm{T} 2$ stimuli in a typical AB task have demonstrated that N1/P1 components - reflecting earlier perceptual analysis - appear intact during the AB (Vogel, Luck, \& Shapiro, 1998), as does the N400 component reflecting semantic analysis (Luck, Vogel, \& Shapiro, 1996; but see Batterink, Karns, Yamada, \& Neville, 2010). However, both the N2 and P3 components are present when $\mathrm{T} 2$ can be consciously accessed, but are suppressed during the AB (Kranczioch, Debener, Maye, \& Engel, 2007; Sergent, Baillet, \& Dehaene, 2005; Vogel et al., 1998; Vogel \& Luck, 2002). The N2 is thought to index a stage responsible for selecting a relevant object among distractors (Kennedy, Rawding, Most, \& Hoffman, 2014; Woodman, Arita, \& Luck, 2009), and P3 is thought to 
a Experiment 1 - T1 Task

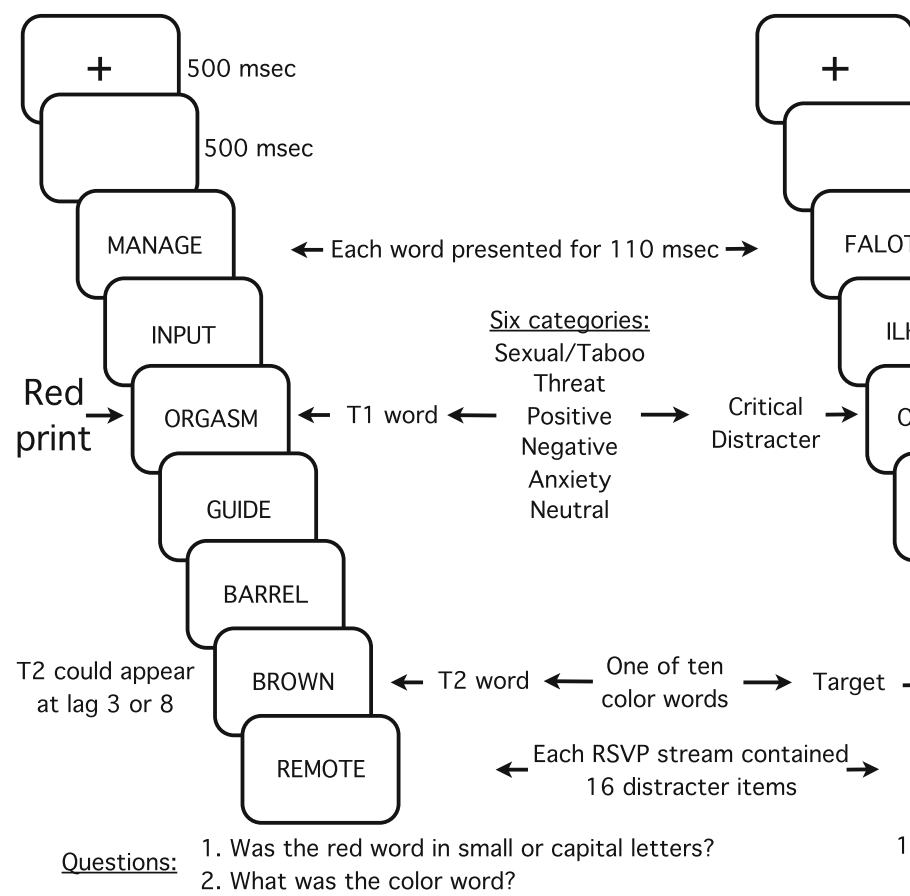

b Experiment 2 - Distracter Task

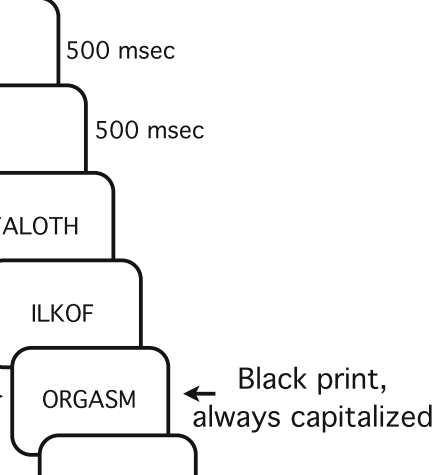

Fig. 1 a The sequence of events within a single trial of the $A B$ task in Experiment 1. b The sequence of events within a single trial of the EIB task in Experiment 2

index the consolidation of the object in working memory (Donchin, 1981; Kranczioch et al., 2007; Sergent et al., 2005; Vogel et al., 1998; Vogel \& Luck, 2002). Additionally, there is evidence that a tradeoff exists between T1 and T2 processing (Kranczioch et al., 2007; Sergent et al., 2005; Shapiro, Schmitz, Martens, Hommel, \& Schnitzler, 2006) as there is a positive correlation between the amplitude of the T1 M300 component (MEG equivalent of the P3) and the magnitude of the AB (Shapiro et al., 2006), and a negative correlation between P3 amplitudes of the first and second RSVP targets (Kranczioch et al., 2007). Indeed, it has been demonstrated that the requirement to attend to $\mathrm{T} 1$ primarily affects the N2 and P3 ERP components that are involved in conscious access to T2 (Sergent et al., 2005). Therefore, the N2 and P3 components have been suggested to index the capacitylimited stage in the $\mathrm{AB}$.

\section{Event-related brain potentials following emotionally arousing stimuli}

Two ERP components are typically reported to differentiate emotionally arousing stimuli from neutral stimuli (see Schupp, Flaisch, Stockburger, \& Junghöfer, 2006, for a review), and interestingly these appear to be the same components whose modulation has been shown to predict the $\mathrm{AB}$ as discussed above. The first is the early posterior negativity (EPN; e.g., Junghöfer, Bradley, Elbert, \& Lang, 2001;
Schacht \& Sommer, 2009a). The EPN is a negativity over temporo-occipital sites that peaks at approximately 200 $300 \mathrm{~ms}$ for picture stimuli (e.g., Junghöfer et al., 2001; Schupp et al., 2007), and at approximately $450 \mathrm{~ms}$ for word stimuli (Schacht \& Sommer, 2009a). The EPN has been observed to be larger for more emotionally arousing stimuli, as compared to less arousing stimuli of a similar valence (Junghöfer et al., 2001; Schupp, Junghöfer, Weike, \& Hamm, 2004). The EPN reliably occurs in response to emotionally arousing words, even when they are presented while participants are engaged in an interference task (Kissler et al., 2009). The EPN has been suggested to index an automatic allocation of visual attention to a stimulus of motivational significance such as stimuli that are emotionally arousing or task relevant so these stimuli can be selected for enhanced encoding (Kissler et al., 2009; Schupp et al., 2006). The timing, scalp distribution, and eliciting conditions of the EPN suggest that it may be the same component referred to outside of the emotion literature as N2; indeed, Schupp et al. (2003a, b) defined the EPN as a modulation of the N2. For the purpose of the current paper, we adopt the hypothesis that the N2 and EPN are two labels for the same component, reflecting the same neurocognitive process, and thus use these labels interchangeably.

The second ERP component that is modulated by emotional stimuli is the late positive potential (LPP). The LPP is a positive increase in amplitude that occurs at centro-parietal areas beginning 
around 300-540 ms and lasting for several hundred milliseconds (Kissler et al., 2009; Schupp et al., 2006). The LPP is considered part of the P3 family, which includes components that reflect the encoding into working memory of stimuli that are task relevant or otherwise capture attention (e.g., Schupp et. al., 2006). The LPP has been variously referred to as the P3, P3b, LPP, and late positive complex in different studies (Kissler et al., 2009; Schupp et al., 2006). The timing and length of the LPP are task and stimulusdependent (e.g., Schacht \& Sommer, 2009a, 2009b; Schupp, Ohman, Junghöfer, et al., 2004); the LPP tends of begin around $540 \mathrm{~ms}$ for emotionally arousing word stimuli (Schacht \& Sommer, 2009a). Similar to the EPN, the LPP is enhanced in response to highly emotionally arousing stimuli, such as erotica or pictures of mutilation, as compared to stimuli of the same valence but lower arousal (Schupp et al., 2000, 2004, 2006).

\section{ERPs following emotion words in the $A B$ and EIB}

Given the ERP patterns associated with processing emotionally arousing stimuli, it may be expected that emotionally arousing $\mathrm{T} 1$ words presented during an AB task would result in enhancement of both the EPN and LPP. Also, given the tradeoff between $\mathrm{T} 1$ and $\mathrm{T} 2$ stage 2 processing suggested to exist for short-lag AB trials (as described above, Kranczioch et al., 2007; Sergent et al., 2005; Shapiro et al., 2006), an enhancement of the EPN and LPP components following a $\mathrm{T} 1$ stimulus should result in an increase in $\mathrm{AB}$ magnitude. Behavioral data demonstrating an increase in $\mathrm{AB}$ magnitude following emotionally arousing $\mathrm{T} 1 \mathrm{stimuli}$ are consistent with this prediction, but no research to date has used ERP techniques to examine these predictions in the $\mathrm{AB}$ paradigm.

A single study (Kennedy, Rawding, Most, \& Hoffman, 2014) collected ERPs during an EIB task in order to examine the mechanisms underlying EIB. In this study, emotionally arousing distractor images or emotionally neutral distractor images were presented prior to single target items within an RSVP stream of filler images. Negative emotional distractor images elicited enhanced N2 (EPN) and P3b (LPP) components relative to emotionally neutral images. Additionally, the amplitude of these components following the distractor stimulus was inversely related to their amplitude following the target stimulus. Finally, the P3b component associated with observing the emotional distractor image was observed to be larger for error trials in which the subsequent target was missed, than for correct trials. This pattern provides electrophysiological evidence of a distractor-target processing tradeoff similar to the T1-T2 processing tradeoff observed in the context of the AB (Kranczioch et al., 2007; Sergent et al., 2005; Shapiro et al., 2006), suggesting that the AB and EIB have similar underlying mechanisms in that they are both a result of central interference in a limited capacity system.

\section{The current study}

The current study asked participants to complete either an AB task with emotionally arousing T1 words (Experiment 1) or an EIB task with an emotionally arousing distractor words (Experiment 2). ERPs time-locked to T1 or the emotional distractor words were collected as participants completed these tasks. To our knowledge, no research has used electrophysiological recording to examine the impact of emotionally arousing T1 words on the in the AB paradigm. Additionally, no research has used ERPs to examine the brain activity during both $\mathrm{AB}$ and EIB tasks with closely matched stimuli and procedures in order to allow a direct comparison of the mechanisms underlying these two tasks.

The behavioral methodology in the current study replicates that of Mathewson et al. (2008). For each of the RSVP tasks, words from six emotion categories were used as T1 or the distractor words (sexual/taboo, positive, negative (sadness), threat, anxiety, neutral). The T1-T2 (AB task) and distractortarget (EIB task) lags were either three items or eight items. Following the RSVP tasks, participants completed an unexpected recognition memory test and provided ratings of valence and arousal for each of the emotion words.

As described above, previous research has observed quite similar patterns of behavioral data resulting from the two tasks used in the current study (Mathewson et al., 2008). It was expected that we would replicate the behavioral results of Mathewson et al. (2008) in this study. Given the similarities in behavioral results resulting from these tasks, and the suggestion that both EIB and the $\mathrm{AB}$ are a result of central interference in a limited capacity system (Kennedy et al., 2014; Kranczioch et al., 2007; Sergent et al., 2005; Shapiro et al., 2006), we expected that emotionally arousing T1 words in the AB task (Experiment 1) would evoke ERP effects similar to those resulting from emotionally arousing distractor words in the EIB task (Experiment 2). More specifically, based on the results of Kennedy et al. (2014), we expected that words from the sexual/taboo category would evoke the largest EPN and LPP components in both tasks, and that these components would demonstrate a tradeoff with T2/target accuracy. It was also expected that, in both tasks, amplitude of the EPN and LPP components resulting from T1/emotional distractors would be positively correlated with word arousal ratings and post-task memory scores, and negatively correlated with T2/ target accuracy at lag 3 , but not lag 8 .

\section{Experiment 1}

\section{Method}

Participants Thirty-one Brock University undergraduate students (22 female) with a mean age of 19.5 years participated in 
this study for course credit or a small monetary payment. They were tested individually in a single session lasting about $4 \mathrm{~h}$. All reported normal or corrected-to-normal vision, English as a first language, and no history of neurological problems. Both experiments reported here received clearance from the Brock University Research Ethics Board and were run in accordance with the approved protocol that conforms to the principles of the Declaration of Helsinki, including obtaining written informed consent from all participants. Two participants were removed from all analyses for their failure to show any clear ERP components for the RSVP task.

Design and stimuli The $\mathrm{AB}$ task used a 6 (emotion condition for T1) X 2 (T1-T2 lag) within-participants design. In each RSVP stream there were two targets. On each trial T1 was a word from one of six emotion categories: positive (e.g., fun, happy, winner), negative words that were sadness-related (e.g., weep, dreary, gloom), threat-related (e.g., scream, cancer, stabbed), words that were either sexual and/or taboo (e.g., fuck, pussy, shit), anxiety-related (e.g., worry, humiliated, disappoint), or emotionally neutral (e.g., jacket, vote, chew). There were 26 words in each emotion category. The T1 emotional word lists were the same as those used by Arnell et al. (2007) and Mathewson et al. (2008) and were adapted originally from stimuli used by Anderson (2005) and McKenna and Sharma (1995). The T1 word was always presented in red, and was presented in capital letters on half of the trials and lowercase letters on the other half of the trials. Each T1 word appeared in uppercase once at each lag, and in lowercase once at each lag. T2 was one of ten words that spelled a color name (blue, green, yellow, orange, white, silver, pink, purple, brown, black) but was always presented in black font, in capitals. Distractor stimuli for the RSVP task were created a priori to be 60 neutral valence and low arousal words from four to seven letters in length. For presentation, distractor words were chosen randomly without replacement for each trial and presented in black, capital letters. All words were presented in 18-point bold Courier New font. The letters subtended approximately $1.4^{\circ}$ of visual angle in height and $3.6^{\circ}-7.2^{\circ}$ in width at an unfixed binocular viewing distance of approximately $40 \mathrm{~cm}$.

Procedure The experiment consisted of three parts: (1) the RSVP task, (2) a surprise recognition memory task where participants were asked to check-off any $\mathrm{T} 1$ words that they recalled seeing in the RSVP task, and (3) a ratings task where participants rated the arousal and valence of each of the $\mathrm{T} 1$ words.

For the RSVP task, participants were instructed to report whether the red $\mathrm{T} 1$ word was presented in uppercase or lowercase letters, and the identity of the $\mathrm{T} 2$ color word. Participants were not told that some of the T1 words would be emotionally charged. They were shown the ten color words, and informed that $\mathrm{T} 2$ would always be from this set, and only these responses would be allowed. Approximately five practice RSVP trials preceded the experimental trials. See Fig. 1a for a graphical depiction of a trial.

Each trial began with the presentation of a black fixation cross in the center of the screen for $500 \mathrm{~ms}$, followed by a 500 ms blank interval before the start of the RSVP stream. Eighteen words, including T1, T2, and 16 distractors, were presented on each trial using RSVP, where each stimulus is presented one-at-a-time in the same spatial location. Each word was presented in the center of a uniform gray screen for $117 \mathrm{~ms}$ with no inter-stimulus interval between words. $\mathrm{T} 1$ was presented in stream position 5 or 8 , and each position was used equally often for each combination of emotion condition and lag. The identity of the T1 word was chosen randomly within each emotion condition with the constraint that each word was shown once in each block of 156 trials, and that each word was presented twice in the lag 3 condition and twice in the lag 8 condition. The identity of the $\mathrm{T} 2$ color word was chosen randomly by the computer with the constraint that each word was used once every 10 trials. T2 was presented either three or eight words after the T1 word, corresponding to 351 or $936 \mathrm{~ms}$ of separation. The levels of the emotion and lag factors varied randomly for each participant, with the constraint that each possible combination of the factors occurred twice every 24 trials. Each participant performed 624 trials in a single session.

One second after the end of each stream, a sentence appeared on the screen prompting participants to press one of two keys indicating whether the $\mathrm{T} 1$ was presented in upper or lower case letters. Immediately after their T1 response a second sentence appeared prompting them to press the labeled key matching the color identity of T2. Accuracy was stressed and responses were not speeded. Participants were asked to minimize their physical movements while viewing the stream, and to refrain from blinking until they saw the first sentence prompting their response after the stream. Two seconds after their button press the fixation cross for the next trial appeared. ERPs were recorded during the RSVP task and were timelocked to the onset of the $\mathrm{T} 1$ stimulus.

Within 3 min of completing the RSVP task, participants were given a surprise recognition memory test. A piece of paper contained a list of all $156 \mathrm{~T} 1$ emotion words plus three word foils from each of the six emotion categories that had not been presented during the RSVP task. All words were presented in alphabetical order. Participants were told that some of the words on the list were presented as red words in the RSVP streams, and that they should check off any words that they remember seeing from the RSVP task. Participants were allowed to go through the list in any order with no time constraints, and could check as many or as few items as they wanted. ERPs were not recorded during the memory test. The number of participants, out of 29 , who checked off each word on the memory checklist was calculated separately for each of the target words and each of the 18 memory foil words to create a memory score for each word. 
These memory scores were then used to calculate the average memory score for each of the emotion conditions and in the correlations. $^{1}$

Following the completion of the memory task, participants then received the ratings task. On each trial one of the 156 words that had been presented as T1 was presented at the center of the computer screen for one second. After one second the word remained on the screen, but the prompt "Valence?" was then added just below. The word and prompt stayed on the screen until the participant gave the word a valence rating. The prompt then changed to "Arousal?" and the prompt and the word remained on the screen until the participant gave the word an arousal rating. A 7-point Likert scale was used for both valence and arousal ratings. The valence scale was anchored by "unpleasant" for the 1 response and "pleasant" for the 7 response, with 4 being "neutral." The arousal scale was anchored by "low" for the 1 response and "high" for the 7 response. Participants used the numbered keys from 1 to 7 to make their response. Participants were asked to make the valence and arousal ratings independently, and to try to use the whole scale. Participants were encouraged to take their time and provide accurate ratings based on their own personal views about the word. The $156 \mathrm{~T} 1$ words were each presented once in random order. ERPs were recorded during the ratings task and were time-locked to the onset of the emotion word on each trial. The Ratings task ERP results are not a focus of the current study and will not be discussed further. Mean valence ratings and mean arousal ratings were calculated for each word by averaging ratings for each word across participants. An absolute valence extremity score was also calculated for each word by subtracting the valence rating for each word from " 4 ," which is the midpoint on the valence scale. In this manner the absolute difference reflects how valent the stimulus was without regard for the direction, as one might expect highly positive and highly negative words to have greater impact.

Apparatus and ERP recordings A desktop PC with 17-in. color monitor, running E-Prime (Psychology Software Tools, Pittsburgh, PA) was used to present stimuli and record behavioral responses. Neuroscan software running on a desktop PC was used to acquire and analyze electroencephalographic (EEG) data recordings from 64 sites (cap by Electrocap International) referenced to linked earlobes. Electro-ocular (EOG) recordings were taken by affixing electrodes to the outer canthi of each eye and the top and bottom of the orbit of both eyes. Signals were amplified with a band-pass of $0.15-30 \mathrm{~Hz}$, and digitized at a rate of

\footnotetext{
${ }^{1}$ Due to a programming creation error, memory scores and ratings were not available for one of the threat words and one of the anxiety words, so these values were calculated for 154 of the $156 \mathrm{~T} 1$ emotion words in Experiment 1 and emotional distractor words in Experiment 2.
}

$500 \mathrm{~Hz}$. ERPs were time-locked to the onset of T1. Epochs were created that began $200 \mathrm{~ms}$ prior to $\mathrm{T} 1$ presentation and ended $1000 \mathrm{~ms}$ after T1 presentation. Eye blink artifacts were corrected using an algorithm implemented in Neuroscan's SCAN software. This algorithm creates a model of the subject-specific blink response by first identifying the maximum deviation in the VEOG channel across all the data (i.e., the largest blink artifact), and then defining as blinks all other events that exceed $10 \%$ of this maximum in the VEOG channel. Transmission coefficient of the blinks is then estimated based on the covariance of the averaged potentials of the ocular channel with the EEG channels, and this is then used to subtract the blink artifact form each channel, on each trial in which a blink was detected. If this correction appeared insufficient for a given trial based on visual inspection then the trial was removed by hand prior to averaging. Trials with incorrect $\mathrm{T} 1$ responses were also removed.

\section{Results}

\section{Behavioral analyses}

Mean differences in RSVP performance Figure 2 shows the mean T2 accuracy (percent correct responses) separately for each T1 emotion condition as a function of the lag between T1 and T2. In both experiments, T2 accuracy was calculated for T1-correct trials only; however, the same data patterns were observed for all analyses when T2 accuracy was not made conditional on T1 accuracy. A repeated measures analysis of variance (ANOVA) was performed on T2 accuracy rates with

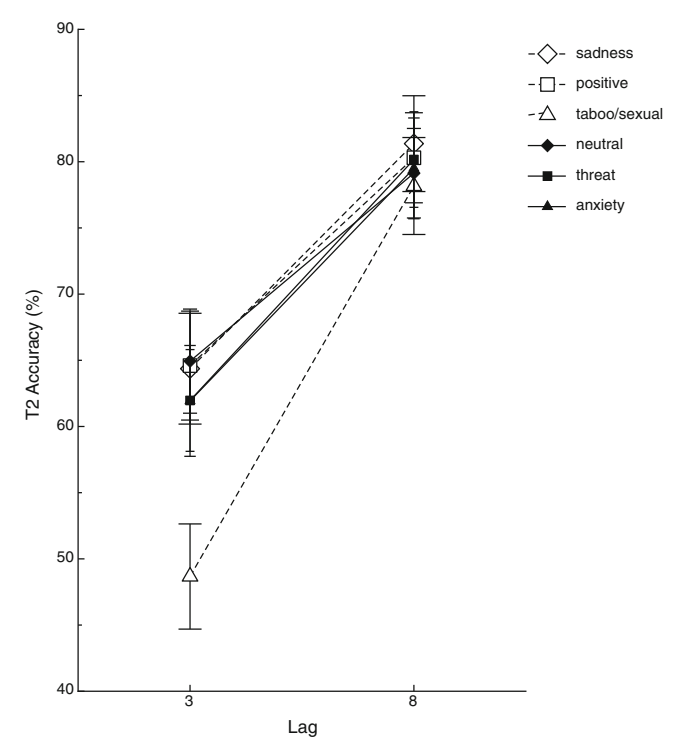

Fig. 2 The mean percentage of correct T2 responses as a function of T1 emotion category and the lag between $\mathrm{T} 1$ and $\mathrm{T} 2$ in the $\mathrm{AB}$ task, Experiment 1. Error bars represent the standard error of the mean 
T1 emotion condition and lag as factors. The ANOVA revealed a significant main effect of lag $[\mathrm{F}(1,28)=56.25$, $\mathrm{p}<$ .001 , partial $\left.\eta^{2}=0.67\right]$ where $\mathrm{T} 2$ accuracy was reduced at lag 3 as compared to lag 8 . There was also a significant main effect of emotion condition $[\mathrm{F}(5,140)=17.79, \mathrm{p}<.001$, partial $\left.\eta^{2}=0.39\right]$. Importantly, there was a significant interaction between lag and $\mathrm{T} 1$ emotion condition $[\mathrm{F}(5,140)=13.97$, $\mathrm{p}$ $<.001$, partial $\eta^{2}=0.33$ ], which resulted from the particularly poor T2 accuracy at lag 3 when taboo/sexual words were presented as $\mathrm{T} 1$.

Based on paired samples t-tests comparing T2 accuracy at lag 3 versus lag 8, a reliable $\mathrm{AB}$ was observed for all $\mathrm{T} 1$ emotion conditions [all $p s<.001]$. Simple-effects analyses using one-way ANOVAs showed no significant difference in T2 accuracy rates as a function of $\mathrm{T} 1$ emotion conditions at lag $8[F(5,140)=1.32$, $p>.26$; partial $\left.\eta^{2}=0.05\right]$. However, a significant difference in T2 accuracy was found at lag $3[F(5,140)=22.21, p<.001$; partial $\left.\eta^{2}=0.44\right]$. Pairwise comparisons with Bonferroni correction showed that at lag $3 \mathrm{~T} 2$ accuracy in the taboo/sexual condition was significantly lower than $\mathrm{T} 2$ accuracy in each of the other emotion conditions [all $p \mathrm{~s}<.001]$. There were no differences in $\mathrm{T} 2$ accuracy between any other conditions [all $p \mathrm{~s}>.91$ ].

$\mathrm{T} 1$ accuracy was $92.3 \%$ (S.E. $=1.2$ ) overall, and did not vary with lag $\left[F(1,28)=1.63, p>.21\right.$, partial $\left.\eta^{2}=0.06\right]$, and there was no significant interaction between lag and emotion condition, $[F$ $<1$,$] . However, T1 accuracy did vary slightly, but significantly,$ across emotion conditions $\left[F(5,140)=3.13, p=.01\right.$, partial $\eta^{2}=$ 0.10]. T1 accuracy was between $92 \%$ and $93 \%$ for each of the emotion conditions except for the taboo/sexual condition where it was $90.9 \%$. Bonferroni-corrected pairwise comparisons showed significant differences in T1 accuracy only between the taboo/sexual condition and the neutral condition, and the taboo/ sexual condition and the anxiety condition $[p s<.05]$.

Memory for T1 words by emotion condition Table 1 shows the average memory score for the words in each emotion condition. A one-way ANOVA showed a significant effect of emotion condition on memory for the target words $[F(5,148)=54.04, p<.001]$. Bonferroni-corrected pairwise comparisons showed that memory for taboo/sexual words was

Table 1 Mean number of participants in Experiment 1 reporting the T1 words as remembered on the surprise recognition memory test as a function of $\mathrm{T} 1$ emotion category

\begin{tabular}{lc}
\hline Emotion Category & Mean word recognition score \\
\hline Sadness & 7.62 \\
Positive & 8.21 \\
Sexual/Taboo & 21.31 \\
Neutral & 6.59 \\
Threat & 10.86 \\
Anxiety & 9.36 \\
\hline
\end{tabular}

significantly higher than memory for words in each of the other emotion conditions [all $p \mathrm{~s}<.001]$. Memory for threat words was also significantly higher than memory for neutral or sad words $(p s<.05){ }^{2}$

Relationships amongst behavioral measures The mean accuracy of T2 at each lag, collapsed across participants, was calculated separately for each T1 emotion word. Correlational analyses were conducted to examine the relationships between arousal ratings, valence ratings, valence extremity scores, word memory scores, and T2 accuracy on trials with a specific emotion word presented as T1. For example, the mean arousal, valence, valence extremity, and memory scores for the word orgasm were examined with respect to T2 accuracy on short and long lag trials where orgasm was presented as T1 (see Table 2). Replicating the pattern found by Mathewson and colleagues (2008), T2 accuracy at lag 3, but not lag 8 , was negatively related to arousal ratings and memory for $\mathrm{T} 1$, and memory for $\mathrm{T} 1$ and $\mathrm{T} 1$ arousal ratings were positively correlated. Therefore, T2 accuracy was lower at lag 3, when T1 was rated as highly arousing and well remembered. However, these relationships were not observed when $\mathrm{T} 2$ was presented at lag 8 .

Mediation analysis If highly arousing words are encoded into memory more often than less arousing words, and this encoding is at the expense of accuracy for $\mathrm{T} 2 \mathrm{~s}$ that were presented soon after T1, then the relationship between arousal and T2 accuracy at lag 3 should be mediated by memory for the words. A simultaneous regression with arousal rating and memory as predictors of T2 accuracy at lag 3 showed that while both arousal and memory were significant predictors of T2 accuracy when entered alone, only memory was a significant predictor of $\mathrm{T} 2$ accuracy at lag 3 when both arousal and memory were entered into the model [semipartial $r=.01$, $p>.77$ for arousal and semipartial $r=-.52, p<.001$ for memory]. These results support a fully mediated model (Baron \& Kenny, 1986) where T1 arousal influences encoding of $\mathrm{T} 1$ into memory at the expense of accuracy for closely trailing T2s (see Fig. 3).

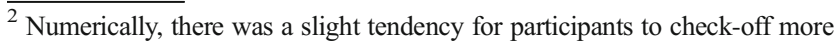
arousing foils. To remove any possible response bias, a net memory score for each word was calculated as the number of hits (the number of checks for each word target) minus the number of false alarms (the average number of checks for the word foils in that emotion category). Memory score analyses were repeated using these net memory scores and again showed that memory for taboo/sexual words was significantly better than memory for words in all other emotion conditions [all $p \mathrm{~s}<.001]$.
} 
Table 2 Zero-order correlations between behavioral and ERP measures for emotional words in Experiment

\begin{tabular}{|c|c|c|c|c|c|c|c|}
\hline & Memory & $\begin{array}{c}\text { T2 } \\
\text { Accuracy } \\
\text { Lag } 3\end{array}$ & $\begin{array}{c}\text { T2 } \\
\text { Accuracy } \\
\text { Lag } 8\end{array}$ & Arousal & Valence & $\begin{array}{c}\text { EPN } \\
: \text { amplitude }\end{array}$ & $\begin{array}{l}\text { LPP } \\
\text { amplitude }\end{array}$ \\
\hline Memory & --- & & & & & : $0.31^{* *}$ & $0.20^{*}$ \\
\hline $\begin{array}{c}\text { T2 } \\
\text { Accuracy } \\
\text { Lag } 3\end{array}$ & $-0.65^{\star *}$ & --- & & & & $-0.23^{*}$ & $-0.28^{\star \star}$ \\
\hline $\begin{array}{c}\text { T2 } \\
\text { Accuracy } \\
\text { Lag } 8\end{array}$ & -0.11 & 0.15 & --- & & & : & -0.09 \\
\hline Arousal & $0.48^{* *}$ & $-0.34^{* *}$ & -0.01 & --- & & $0.25^{*}$ & $0.18^{*}$ \\
\hline Valence & 0.01 & -0.03 & -0.02 & $0.39^{* *}$ & --- & -0.03 & 0.05 \\
\hline $\begin{array}{l}\text { Valence } \\
\text { Extremity }\end{array}$ & -0.11 & $0.20^{\star}$ & 0.11 & $0.39^{\star \star}$ & $-0.17^{*}$ & : $\quad 0.06$ & 0.07 \\
\hline
\end{tabular}

\section{ERP analyses}

Effects of T1 word type Electrode $\mathrm{Pz}$ was used for all figures and analyses, as activation related to the LPP component was of particular interest, and the P3 family is often maximal in centro-parietal areas (e.g., Donchin, 1981; Schupp et al., 2006). In previous research, the early posterior negativity (EPN) component has been observed over lateralized temporo-occipital sites (Schupp et al., 2006). However, examination of topographical maps of the data in the present study, shown in Fig. 4, revealed that the EPN was present over a large area of the scalp, centered over centro-parietal electrodes. As a result, $\mathrm{Pz}$ was deemed a suitable site for measurement of both the EPN and LPP in the current study.

Only T1-correct trials were included in the averages. Figure $5 \mathrm{a}$ shows the grand average waveforms from the RSVP task separately for each emotion condition, timelocked to the presentation of the T1 emotion word. Figure $5 \mathrm{~b}$ shows grand average difference waves from five emotion conditions once the average waves from neutral trials were subtracted out. ${ }^{3}$

The first goal was to look for potential amplitude differences in the EPN and LPP for different emotion conditions. However, accurate estimation of individual components for each participant is difficult with ERPs collected during RSVP due to the noise that results from each item in the stimulus stream producing ERPs that overlap in time. One solution is to examine the difference waves by subtracting out activation from the neutral condition - as shown in Figs. 4 and 5b. However, we were interested in statistically comparing ERPs to the neutral condition, which would not be possible with neutral waves subtracted out. Furthermore, we were also interested in looking for potential differences amongst emotion conditions outside the EPN and LPP windows.

\footnotetext{
${ }^{3}$ The continuous presentation of stimuli in the RSVP stream results in numerous ERP components in addition to the ones from T1 and T2. Because these additional components are likely to be highly similar for each of the emotion conditions, the creation of difference waves by subtracting the activation on neutral trials from activation on the other emotion conditions substantially reduces the noise and allows for an easier examination of the $\mathrm{T} 1$ waveform differences across the remaining emotion conditions.
} 


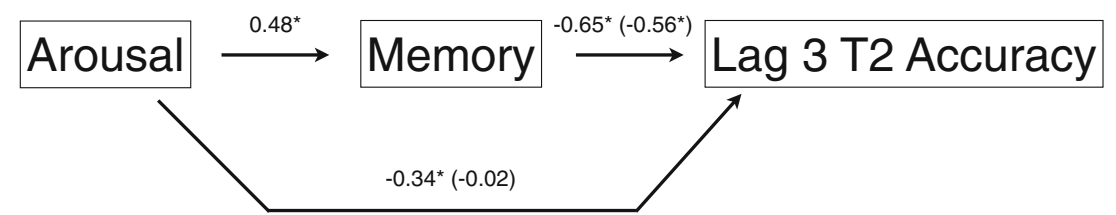

Fig. 3 In Experiment 1, the relationship between arousal ratings of T1 words and accuracy of responses to subsequent T2 items at short lags was fully mediated by memory for T1 words on a surprise post-RSVP task memory test

Therefore, each participant's average post-T1 waveform for each of the emotion conditions was divided into 10-ms intervals, and each of the intervals from 0 to $1,000 \mathrm{~ms}$ post-T1 were used to compare the amplitude across emotion conditions in a series of $t$ tests. To account for experiment-wise alpha inflation, an alpha level of .005 was used as the significance cut-off and at least two consecutive 10-ms intervals were required to have a $p$-value
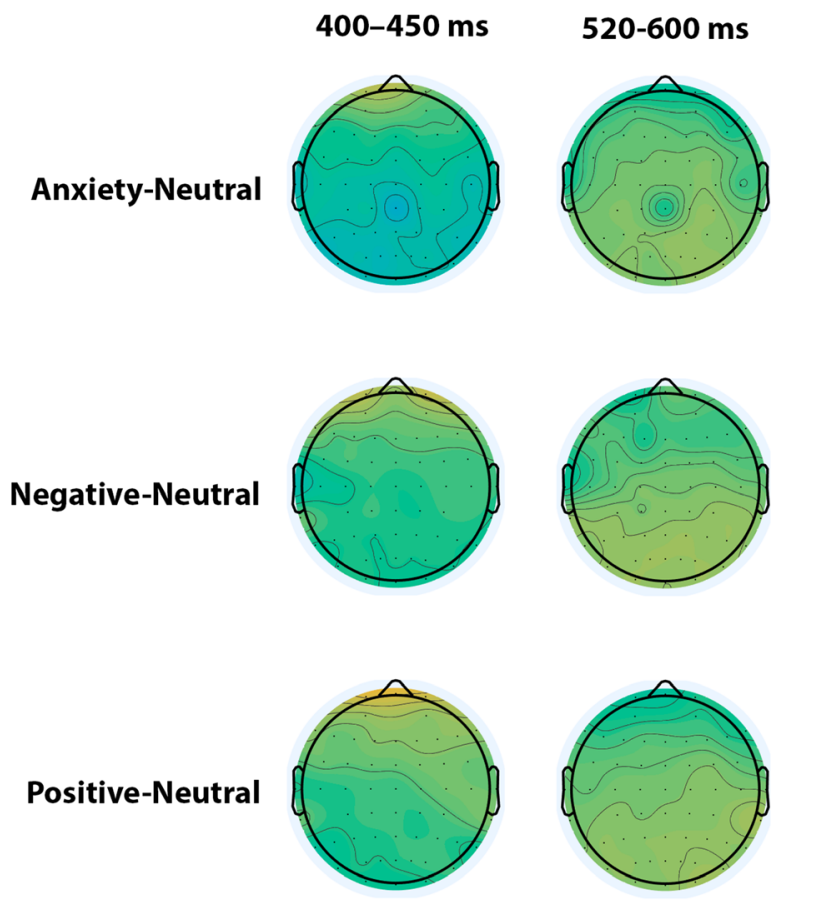

Sexual/Taboo-Neutral
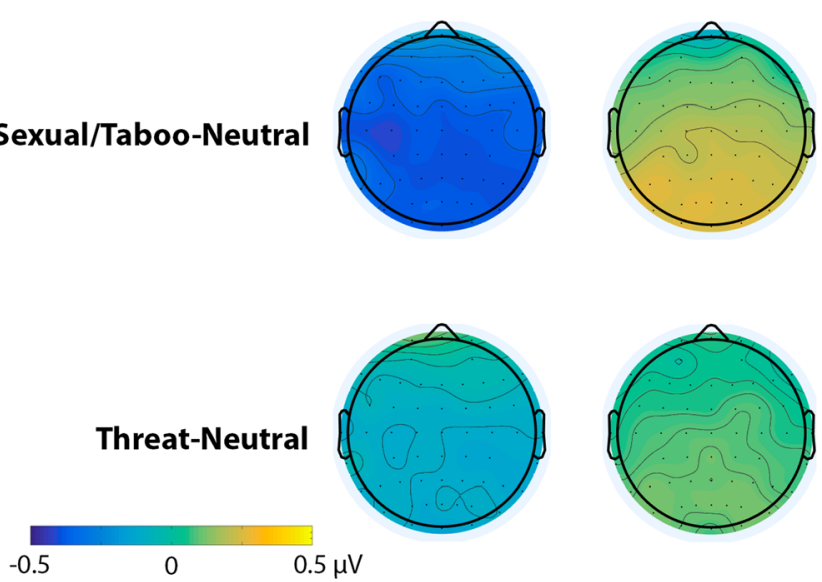

Fig. 4 Topographic maps showing scalp distributions of differences between each experimental $\mathrm{T} 1$ stimulus type relative to the neutral $\mathrm{T} 1$ words during the EPN (left) and LPP (right) time windows less than the .005 level. Paired $t$-tests revealed no significant amplitude differences at any time points between waveforms from the neutral, threat, sadness, positive and anxiety conditions (each compared to each other). ${ }^{4}$ In contrast to the null effects for other emotion categories, there were several differences between the waveform for the taboo/sexual condition and other conditions.

$T$-tests revealed a significantly increased negativity for taboo/ sexual words relative to all other emotion categories from about 400 to $450 \mathrm{~ms}$ (see Fig. 5b). This was confirmed with an analysis of items means, ${ }^{5} p<.01$. In the subsequent discussion, we will refer to this effect as the EPN. The amplitude of the taboo/sexual waveform was also significantly more positive than the neutral waveform from 520 to $600 \mathrm{~ms}$ post-T1 (see Fig. 5b). This was also confirmed with an analysis of item means, $p<.01$. In subsequent discussion, we will refer to this effect as the LPP. Although both the EPN and LPP appear later than has been observed in ERP studies using picture stimuli (see timing suggested by Schupp et al., 2006), this timing is expected for word stimuli, and is similar to that observed for word stimuli in previous research (Schacht \& Sommer, 2009b). No other significant differences in amplitude between the taboo/ sexual waveform and any other waveforms were noted. Figure $5 \mathrm{~b}$ also shows an enhanced positivity for sexual/taboo words just prior to $800 \mathrm{~ms}$. However, this difference was not significant with the alpha correction used to control Type I error rates. Given its late duration, this positivity likely represents modulation of T2 processing when T2 was presented at short lags. This is discussed below and in Experiment 2 where there is further analysis and discussion.

T1-locked ERPs were compared for trials where $\mathrm{T} 2$ was correct and trials where $\mathrm{T} 2$ was incorrect, separately for each lag (see Fig. 6). ${ }^{6}$ At long lags there was expected to be no difference in $\mathrm{T} 1$ ERPs as a function of accurate $\mathrm{T} 2$ detection, as $\mathrm{T} 1$ processing

\footnotetext{
${ }^{4}$ The lack of seemingly random differences that were not hypothesized presumably reflecting Type I errors - suggests that the present strategy was relatively effective at controlling alpha.

${ }^{5}$ In these analyses of item means, ERP amplitude was collapsed across participants separately for each word to get means for each word. These were used to confirm effects observed with the more typical participant analyses where ERP amplitude was collapsed across words separately for each participant to get condition means for each participant. In the same way that typical participant analyses attempt to ensure that mean differences are somewhat generalizable across participants, item analyses help to ensure that mean differences are somewhat generalizable across items (in this case words).

${ }^{6}$ The ERPs here were collapsed across emotion category as there were not enough trials in each bin for most participants when data were broken down into each combination of lag, correct/incorrect, and emotion category. However, visual inspection of the grand average waves for the sexual word conditions shows the same pattern as above in both experiments, and supports the same conclusions reported as when the data are collapsed across emotion category.
} 


\section{a Individual Condition Waveforms}

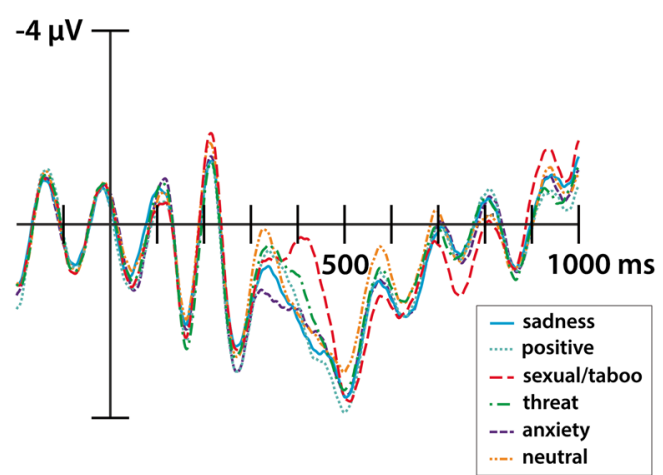

b Difference waves (each vs. neutral)

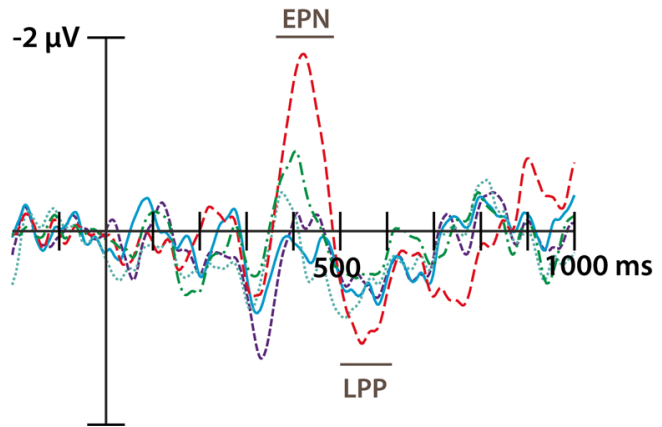

Fig. 5 a Experiment 1 grand average ERPs time-locked to T1 recorded from the midline parietal electrode site $(\mathrm{Pz})$, by $\mathrm{T} 1$ emotion category. $\mathbf{b}$ Experiment $1 \mathrm{~T} 1$-locked difference waveforms obtained by subtracting the neutral emotion condition waveform from each of the other T1 emotion condition waveforms at electrode site $\mathrm{Pz}$

would be over by the time $\mathrm{T} 2$ was presented. However, a tradeoff was expected between $\mathrm{T} 1$ resources and $\mathrm{T} 2$ performance at short lags, such that T1 EPN and LPP amplitudes were expected to be enhanced for T2-incorrect trials relative to T2-correct trials. As expected, paired sample-tests, corrected as above, showed no differences in T1-locked ERPs at any time point when comparing T2correct and -incorrect trials at lag 8. However, at lag 3, the LPP from 500 to $600 \mathrm{~ms}$ post-T1 was significantly larger (more positive) on T2-incorrect trials than on T2-correct trials, suggesting that trials with a large LPP to T1 resulted in poorer T2 performance; i.e., the expected tradeoff. Interestingly, there were no significant amplitude differences for T2-correct and -incorrect short-lag trials during the T1 EPN time window.

At lag 3, T2-correct trials did show a significantly greater negativity than T2-incorrect trials from 650 to $760 \mathrm{~ms}$ post-T1, presumably reflecting the EPN that was present for T2 when it was detected, but not when missed due to an $\mathrm{AB}$ effect. Similarly, at lag 3 T2-correct trials showed a significantly greater positivity than T2-incorrect trials from 870 to $940 \mathrm{~ms}$ post$\mathrm{T} 1$, presumably reflecting the LPP that was present for T2 on $\mathrm{T} 2$-correct trials, but attenuated on T2-incorrect trials.

Relationships between behavioral and ERP measures The mean T1-EPN was estimated individually for each word by a Short Lag (3 items)
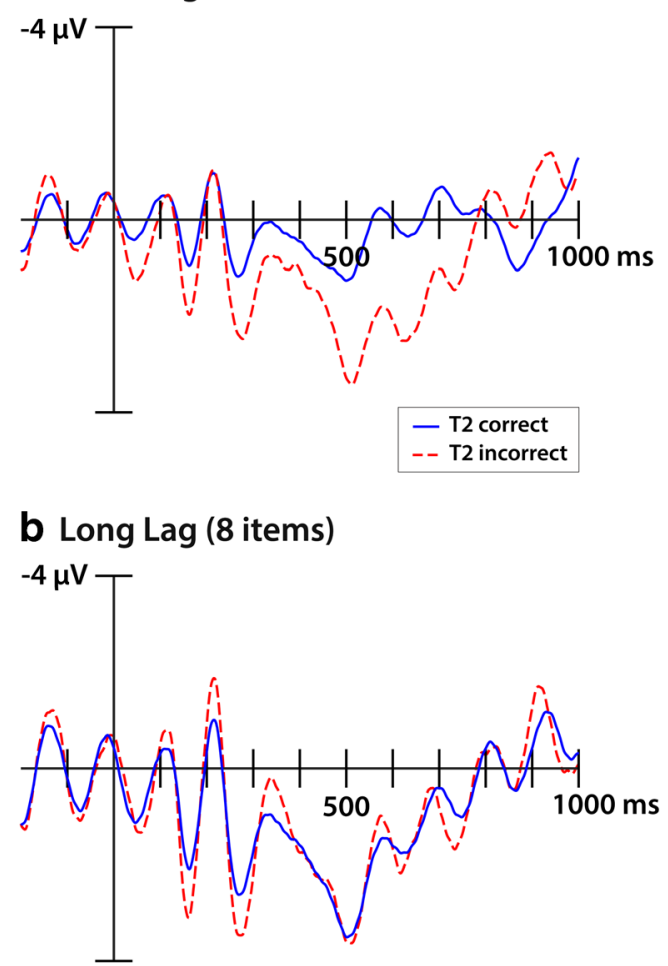

Fig. 6 Experiment 1, T1-locked, grand average waveforms at electrode site $\mathrm{Pz}$ as a function of whether T2 was correctly or incorrectly identified. Panel a shows data from lag 3 trials, and panel b from lag 8 trials

taking the mean area of the component with the largest negative peak between 350 and $500 \mathrm{~ms}$ post-T1 in the grand average waveform averaged across participants for each word. The mean T1-LPP was estimated individually for each word by taking the mean area of the component with the largest positive peak between 500 and $600 \mathrm{~ms}$ post-T1 in the grand average waveform averaged across participants for each word (see above for justification for these time windows). Although mean area was used as a measure of component amplitude for all correlations, the same pattern of significant relationships was also observed when component amplitude was estimated using peak amplitude or when using the summed area of the component. Correlations between the EPN and LPP individually for each $\mathrm{T} 1$ word and behavioral measures for those words are provided in Table 2. It is noteworthy that the amplitude of both of the ERP components was significantly positively associated with memory and arousal ratings for $\mathrm{T} 1$ words, and negatively associated with $\mathrm{T} 2$ accuracy at lag 3 but not lag $8 .^{7}$

\footnotetext{
${ }^{7}$ In addition to these simple correlation analyses, further multiple regressions were conducted in order to examine the independent contributions of arousal, valence, and valence extremity to variability in memory, T2 lag 3 accuracy and both ERP components. In each case, arousal remained a significant predictor once valence and valence extremity were taken into account.
} 
Predicting T2 accuracy In the above analyses, arousal ratings, memory performance, and LPP and EPN amplitudes each predicted T2 accuracy at lag 3 , but not at lag 8 . To see how much total variability in T2 accuracy could be explained, these predictors were entered as simultaneous predictors of lag $3 \mathrm{~T} 2$ accuracy in a multiple regression. Results showed that, as a group, these predictors explained almost half $(45.5 \%)$ of the variability in $\mathrm{T} 2$ accuracy at lag $3(\mathrm{R}=0.675, F(4,146)=$ $30.39, p<.001$ ). Both memory performance (semipartial $r=$ $-.48, p<.001$ ) and LPP amplitude (semipartial $r=-.16, p=$ .009) contributed significant unique variability over and above that contributed by the other predictors. When the same regression was performed on T2 accuracy at lag 8, a nonsignificant $2.3 \%$ of the variability in T2 accuracy was explained $(\mathrm{R}=.153, F<1, p=.47)$, and there were no significant unique predictors.

\section{Discussion}

The $\mathrm{AB}$ was equal in size for all emotion conditions except the taboo/sexual condition, where a larger $\mathrm{AB}$ was observed. These results replicate those of Mathewson et al. (2008) when using emotion words as T1s. Also replicating Mathewson et al. (2008), we found that memory was better for the taboo/sexual words than for other emotion words and enhanced memory for a $\mathrm{T} 1$ word was associated with lower accuracy when $\mathrm{T} 2$ was presented in the lag 3 position. We further replicated Mathewson et al.'s (2008) finding that higher word arousal ratings predicted enhanced memory for the word and lower accuracy for subsequent targets, and that the relationship between arousal and T2 accuracy was mediated by memory performance (Fig. 3). This pattern suggests that arousing T1s are better encoded into memory than other $\mathrm{T} 1 \mathrm{~s}$, and that this increase in encoding reduces accuracy for subsequent T2s.

As expected, taboo/sexual T1s showed an enhanced EPN relative to all other emotion conditions. Taboo/sexual words were also the only word type to show an enhanced LPP when compared to emotionally neutral words. There was also a tradeoff between the amplitude of the LPP to T1 and the LPP to T2, where the T1 LPP was larger for incorrect T2 trials compared to correct T2 trials at short lags, and the LPP (and EPN) to T2 were greater on correct $\mathrm{T} 2$ short lag trials. Correlational analyses demonstrated that EPN and LPP amplitudes increased with arousal ratings, were associated with better memory for $\mathrm{T} 1$ words, and were negatively related to accuracy for T2s at short the lag, but not at long lags. This pattern suggests that: (1) there was increased activation for arousing T1s, (2) this increased activation enhanced their encoding into memory, and/or resulted from their encoding into memory, and (3) this enhanced encoding came at the expense of $\mathrm{T} 2$ encoding at short lags.
Experiment 1 is the first study to provide evidence that arousing $\mathrm{T} 1$ words in the $\mathrm{AB}$ achieve enhanced processing in stages associated with attentional selection of a target among distractors (N2/EPN; Kennedy et al., 2014; Woodman et al., 2009) and consolidation in working memory (LPP; Sergent et al., 2005; Vogel \& Luck, 2002; Vogel et al., 1998). This is despite the fact that the semantics of the T1 words were irrelevant for the lowercase/uppercase font decision that was the T1 task. Furthermore, degree of enhancement of $\mathrm{T} 1$ word processing at these stages, operationalized as EPN and LPP amplitude associated with the word, was related to the magnitude of the $\mathrm{AB}$ that followed the $\mathrm{T} 1$ word and how well those $\mathrm{T} 1$ words were remembered.

\section{Experiment 2}

As described in the Introduction, arousing words have also been shown to capture participants' attention and set off an EIB effect when they are presented as to-be-ignored distractors in an RSVP stream. Experiment 2 examined whether similar ERP results to those in Experiment 1 are found when emotion words are presented as to-be-ignored distractors but all other experimental factors remain the same.

\section{Methods}

Participants Thirty Brock University male and female undergraduate students participated in this study for credit towards a course or small monetary payment. They were tested individually in a single session lasting approximately $4 \mathrm{~h}$. All reported normal or corrected to normal vision, English as a first language, and no history of neurological problems. None had participated in Experiment 1.

Procedure, apparatus, and stimuli Experiment 2 was identical to Experiment 1 with the following exceptions (see Fig. 1b). The word that served as T1 and was presented in red in Experiment 1, was now presented in black, and always capitalized - the same as all other stimuli in the stream. For Experiment 2, this word will be referred to as the emotional distractor since distractor emotion words continued to be presented in this location within RSVP, but they were not taskrelevant. The uppercase/lowercase task was removed and participants performed only the color word identification task on each trial - previously the T2 task, but now the only task. As in Arnell et al. (2007), the filler words that were not the emotional distractor or the target were changed from 60 neutral words to 60 non-words of the same length so that even emotionally neutral distractor words could be differentiated from fillers. The use of non-words as RSVP fillers allowed the emotional distractor to be equally novel from the fillers aside from emotion because non-words are not associated with any 
semantic meaning (Arnell et al., 2007). The non-words obeyed all orthographic rules of English and included no pseudohomophones. Participants were not told about the emotion word distractors. They were told to identify the written color word on each trial, and to ignore all other stimuli. One second after the end of the RSVP stream a sentence appeared asking them to report the color word. Participants made their responses using the same keys as Experiment 1. Participants were instructed to guess if they were unsure of the identity of the color word. There were no time restrictions placed on the participants as they made their responses. During the recognition memory test, participants were simply told to check off any words they remembered seeing anywhere in the RSVP streams.

\section{Results}

\section{Behavioral analyses}

Mean differences in RSVP performance Figure 7 shows the mean target accuracy separately for each emotion condition as a function of the lag between the emotional distractor and the target. A repeated measures ANOVA was performed on target accuracy with emotion condition and lag as factors. There was a significant main effect of lag $[F(1,29)=26.63, p<.001$, partial $\left.\eta^{2}=0.48\right]$ where accuracy was reduced at lag 3 compared to lag 8 , and a significant main effect of emotion condition $\left[F(5,145)=8.40, p<.001\right.$, partial $\left.\eta^{2}=.23\right]$. Importantly, there was a significant interaction between lag and emotion condition $\left[F(5,145)=6.30, p<.001\right.$, partial $\eta^{2}$ $=0.18]$, resulting from the greater EIB observed when the distractor was a taboo/sexual word.

A significant EIB effect was observed when the emotional distractor was taboo/sexual $[t(29)=5.52, p<.001]$, sad $[t(29)$ $=3.81, p=.001]$, positive $[t(29)=2.23, p<.05]$, and threatening $[t(29)=2.43, p<.05]$. Distractors that were neutral or anxious in nature did not show a significant EIB effect $[p$ s $>$ .24]. A one-way ANOVA showed no reliable difference in target accuracy rates across the emotion conditions at lag 8 $\left[F(5,145)=1.30, p>.26\right.$, partial $\left.\eta^{2}=0.04\right]$. However, a reliable difference in target accuracy was found at lag 3 $\left[F(5,145)=10.79, p<.001\right.$, partial $\left.\eta^{2}=0.27\right]$. Pairwise comparisons with Bonferroni correction showed that target accuracy at lag 3 in the taboo/sexual condition differed from target accuracy at lag 3 in each of the other emotion conditions [all $p$ s $<.001]$. There were no differences in target accuracy for any other conditions [all $p s>.99$ ].

Memory for distractor words by emotion condition A oneway ANOVA was used to compare memory scores for each emotion condition (see Table 3 for means). The ANOVA showed a significant effect of emotion condition $[F(5,147)=$ 23.96, $\left.p<.001, \eta^{2}=0.45\right]$. Bonferroni-corrected pairwise

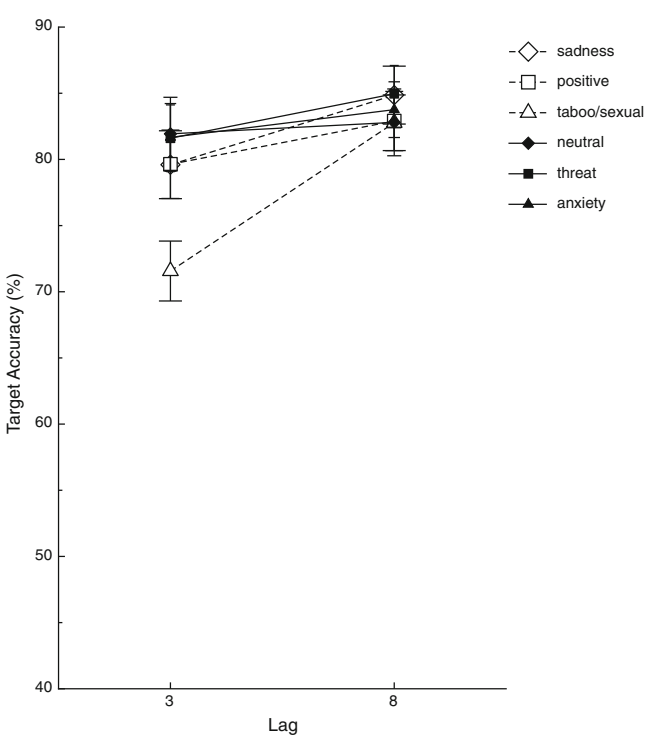

Fig. 7 The mean percentage of correct target responses as a function of emotional distractor emotion category and the lag between the distractor and the target in the EIB task used in Experiment 2. Error bars represent the standard error of the mean

comparisons showed that memory for taboo/sexual words $(\mathrm{M}=11.15)$ was significantly better than memory for words in each of the other emotion conditions $[\mathrm{M}=3.65$ to 5.50 , all $p \mathrm{~s}<.001]$. There were no differences in memory for target words between any other emotion conditions [all $p \mathrm{~s}>.23$ ]. ${ }^{8}$

Correlations amongst behavioral measures For each of the emotion words, mean valence ratings, arousal ratings, valence extremity scores, memory scores, and target accuracy rates were calculated and correlated as in Experiment 1 (see Table 4 for correlations). As in Experiment 1, target accuracy at lag 3 , but not lag 8 , was negatively related to arousal ratings and memory for the emotional distractor words and high arousal ratings predicted better memory.

Mediation analysis A simultaneous regression with arousal rating and memory as predictors of target accuracy at lag 3 showed that while both arousal and memory were significant predictors of target accuracy when entered alone, only memory was a significant predictor of target accuracy at lag 3 when both arousal and memory were entered simultaneously into the model [semipartial $r=-.07, p>.34$ for arousal and semipartial $r=-.42, p<.001$ for memory]. Again, these results support the idea that highly arousing distractors are encoded into memory preferentially, at the expense of accuracy for targets presented soon after them (see Fig. 8).

\footnotetext{
${ }^{8}$ As in Experiment 1, memory score analyses were also conducted using net memory scores, controlling for false alarms. Again, memory for taboo/sexual words was significantly better than memory for words in all other emotion conditions [all $p \mathrm{~s}<.004$ ] with no other significant differences between emotion conditions.
} 
Table 3 Mean number of participants in Experiment 2 reporting the emotional distractor words as remembered on the surprise recognition memory test as a function of emotional distractor emotion category

\begin{tabular}{lc}
\hline Emotion Category & Mean word recognition score \\
\hline Sadness & 3.77 \\
Positive & 4.46 \\
Sexual/Taboo & 11.15 \\
Neutral & 3.65 \\
Threat & 5.50 \\
Anxiety & 4.76 \\
\hline
\end{tabular}

\section{ERP analyses}

Effects of emotional distractor word type Figure 9a and b show the grand average waveforms at site $\mathrm{Pz}$ time-locked to the presentation of the emotional distractor emotion word, separately for each emotion condition. Figure 9a shows the raw grand average waveforms, and Fig. 9b shows grand average difference waves from five emotion conditions once the average waves from neutral trials were subtracted out.

Potential amplitude differences in the different emotion conditions were examined across the waveforms using alpha-corrected $(p<.005)$ t-tests as in Experiment 1 . The taboo/sexual condition had a significantly increased EPN (from 400 to $450 \mathrm{~ms}$ ) relative to all emotion categories except the threat and anxiety conditions. This replicated the findings of Experiment 1 except that the positive and threat conditions also showed significantly increased EPNs relative to the neutral condition (at approximately $400 \mathrm{~ms}$; see Fig. 9b). There were no other significant amplitude differences during this timeframe. Analysis of item means showed that the negativity in the taboo/sexual condition around $400 \mathrm{~ms}$ was significantly larger than in the neutral condition $[p<.03]$, but there were no other significant differences between any emotion conditions in this timeframe.

Following the EPN, the taboo/sexual waveform was significantly more positive than the sadness, positive, threat, and anxiety waveforms from 700 to $740 \mathrm{~ms}$. The taboo/sexual waveform amplitude did not differ from the neutral condition, and there were no other significant amplitude differences between any conditions in the analysis of participant means. However, analysis of item means showed a significantly larger positivity for the taboo/sexual waveform relative to each of the other emotion condition waveforms, at $550 \mathrm{~ms}$ (all $p \mathrm{~s}<.030$ ).

Thus two enhanced positivities were observed for the taboo/sexual condition in this experiment: one at $550 \mathrm{~ms}$, and one from 700 to $740 \mathrm{~ms}$. Note that the increased positivity from 700 to $740 \mathrm{~ms}$ was much later than the LPP in Experiment 1, and late enough that it is unlikely to represent the LPP to the emotional distractor here. In Experiment 1 the LPP was present between 520 and $600 \mathrm{~ms}$, so the significantly greater positivity found at $550 \mathrm{~ms}$ in the item analysis for Experiment 2 - observable in Fig. 9b - likely reflects the LPP in this experiment, which was presumably smaller due to the task in this experiment not requiring memory encoding of irrelevant distractor words. This would also be consistent with the lower number of words marked as remembered on the memory checklist in Experiment 2 where the target was task-irrelevant and less salient - compared to the number of items reported as remembered in Experiment 1 where the target was task-relevant and salient by virtue of its color.

We did note the enhanced positivity for taboo/sexual words from 700 to $740 \mathrm{~ms}$ in Experiment 1, but it fell short of significance there until trials were separated into correct versus incorrect trials at short and long lags where it appeared to reflect a reduced ERP to T2 on incorrect T2 short lag trials relative to correct T2 short lag trials. Kennedy et al. (2014) observed a posterior positivity from about 500-600 ms postdistractor at lateral posterior sites, that was larger following negative distractors than neutral distractors when using long lag trials that ensured the positivity was distractor-related, not target-related. Kennedy et al. suggested that this posterior positivity could be a $\mathrm{P}_{\mathrm{D}}$ component that may have resulted from greater difficulty disengaging attention from the emotional distractor. Thus, it is possible the 700-740 ms positivity observed here reflects this also. If so, then it should be evident even on long lag trials, whereas, only short-lag trials should show the effect if it is related to target, not distractor, processing.

Visual inspection of raw ERP waves in the 700-740 ms time period in Experiment 2 suggests that the greater positivity in the sexual/taboo condition was in fact due to a reduced negative-going component for taboo/sexual items relative to other item types in this time window (see Fig. 9a); it is notable that this is the time period when short-lag target items were presented. This timing suggests that this enhanced 700$740 \mathrm{~ms}$ positivity may also be interpreted as a reduced target item EPN on short-lag trials.

This interpretation is supported by the likely timing of a target item EPN (351 ms lag between emotional distractor and target + $400 \mathrm{~ms}$ for EPN), and was supported by further analyses that were split by short and long lag. These split-lag analyses allow further consideration of whether the late enhanced positivity described above was a result of a reduced target item EPN. If this component is a reduced target item EPN, it should only be present on short lag trials, not long lag trials, due to the timing of the target item presentation. Indeed, in these split-lag analyses, the EPN and LPP to the target item were clearly visible at the short lag, but not the long lag. Statistical analyses, using multiple $t$-tests in 10 -ms bins, as reported above in Experiment 1, provided evidence of this late enhanced positivity for taboo/sexual items for short lag trials (relative to threat and positive items) from 720 to $750 \mathrm{~ms}$. No 
Table 4 Zero-order correlations between behavioral and ERP measures for emotional words in Experiment 2

\begin{tabular}{|c|c|c|c|c|c|c|c|}
\hline & Memory & $\begin{array}{c}\text { Target } \\
\text { Accuracy } \\
\text { Lag } 3\end{array}$ & $\begin{array}{c}\text { Target } \\
\text { Accuracy } \\
\text { Lag } 8\end{array}$ & Arousal & Valence & $\begin{array}{l}\text { EPN } \\
: \text { amplitude }\end{array}$ & $\begin{array}{c}\text { LPP } \\
\text { amplitude }\end{array}$ \\
\hline Memory & -- & & & & & : $0.17^{*}$ & $0.28^{* *}$ \\
\hline $\begin{array}{c}\text { Target } \\
\text { Accuracy } \\
\text { Lag } 3\end{array}$ & $-0.56^{\star \star}$ & $\cdots$ & & & & $-0.18^{*}$ & $-0.24^{* *}$ \\
\hline $\begin{array}{c}\text { Target } \\
\text { Accuracy } \\
\text { Lag } 8\end{array}$ & -0.11 & 0.11 & --- & & & 0.01 & -0.02 \\
\hline Arousal & $0.42^{* *}$ & $-0.29^{\star \star}$ & -0.09 & --- & & : $0.20^{*}$ & $0.22^{\star \star}$ \\
\hline Valence & 0.02 & -0.11 & -0.15 & $0.57^{* *}$ & --- & : 0.03 & 0.02 \\
\hline $\begin{array}{l}\text { Valence } \\
\text { Extremity }\end{array}$ & -0.04 & 0.1 & 0.09 & $0.24^{* *}$ & $-0.29^{* *}$ & 0.02 & -0.01 \\
\hline
\end{tabular}

significant differences in this time period between any waveforms were observed on long lag trials.

Emotional distractor-locked ERPs were also compared for trials where the target response was correct and trials where the target response was incorrect, separately for each lag (see Fig. 10ab). At long lags there was expected to be no difference in emotional distractor ERPs as a function of target correct/incorrect, as processing of the emotional distractor would be over by the time the target was presented. However, a tradeoff was expected between emotional distractor ERP amplitude and target performance at short lags, such that emotional distractor EPN and LPP amplitudes were expected to be enhanced for target-incorrect trials relative to target-correct trials. As expected, paired samples-tests, corrected as above, showed no differences in emotional distractor-locked ERPs at any time point when comparing target correct and incorrect trials at lag 8. However, at lag 3, target incorrect trials showed significantly greater positive amplitude than target correct trials from 570 to $650 \mathrm{~ms}$ postemotional distractor, suggesting that trials with a large LPP to the emotional distractor resulted in poorer target performance, as expected. Again, there were no significant amplitude differences for target correct and incorrect short-lag trials during the EPN time window.

As in Experiment 1, at lag 3, target correct trials showed significantly greater positive amplitude than target

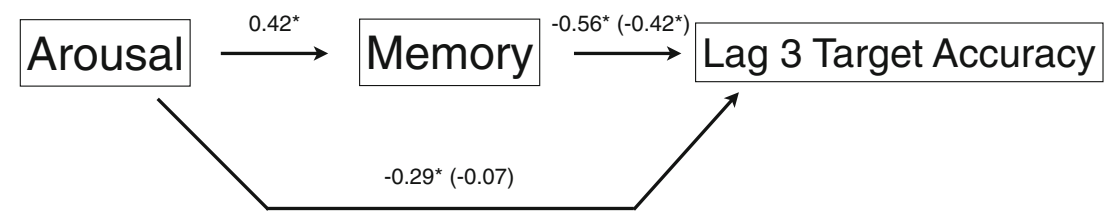

Fig. 8 In Experiment 2, the relationship between arousal ratings of emotional distractor words and accuracy of responses to subsequent target items at short lags was fully mediated by memory for emotional distractor words on a surprise post-RSVP task memory test 


\section{a Individual Condition Waveforms}

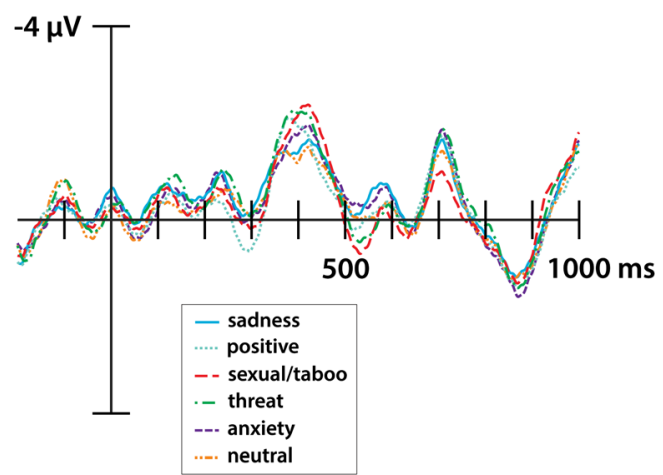

b Difference waves (each vs. neutral)

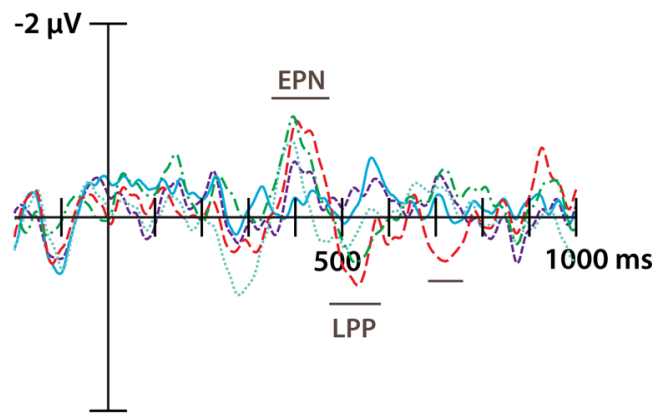

Fig. 9 a Grand average ERPs time-locked to emotional distractor items (in Experiment 2) recorded from the midline parietal electrode site (Pz), split by distractor emotion category. b Experiment 2 emotional distractorlocked difference waveforms obtained by subtracting the neutral emotion condition waveform from each of the other emotional distractor emotion condition waveforms at site $\mathrm{Pz}$

incorrect trials from 650 to $750 \mathrm{~ms}$ post-emotional distractor, consistent with Experiment 1 and the conclusion that EIB results in an attenuated EPN to short lag targets. Target correct trials also showed a significantly greater positive amplitude than target incorrect trials from 830 to $930 \mathrm{~ms}$ post-emotional distractor, which presumably reflects the greater LPP observed to correct versus incorrect targets, as in Experiment 1.

\section{Relationships between behavioural and ERP measures As} in Experiment 1, correlations were examined between behavioral measures and the EPN and LPP amplitudes to the emotional words by estimating these measures individually for each word (see Table 4). As in Experiment 1, amplitude of the distractor EPN component was estimated as the mean area of the component with the largest negative peak between 350 and $500 \mathrm{~ms}$ post-emotional distractor in the grand average waveform averaged across participants for each word. The mean T1-LPP was again estimated individually for each word by taking the mean area of the component with the largest positive peak between 500 and $600 \mathrm{~ms}$ post-T1 in the grand average waveform averaged across participants for each word.

\section{a Short Lag (3 items)}

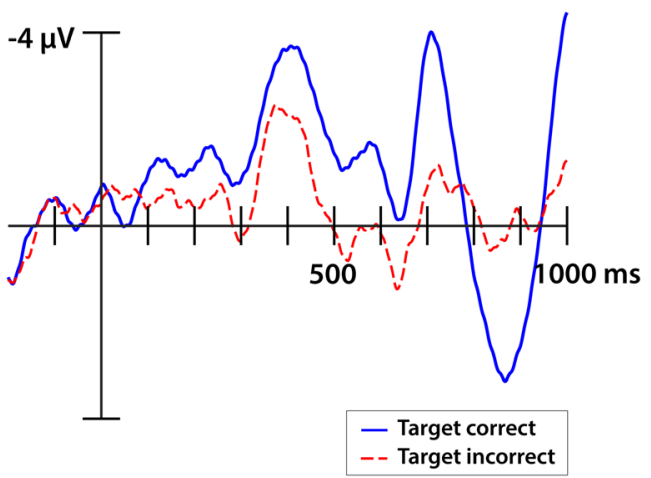

b Long Lag (8 items)

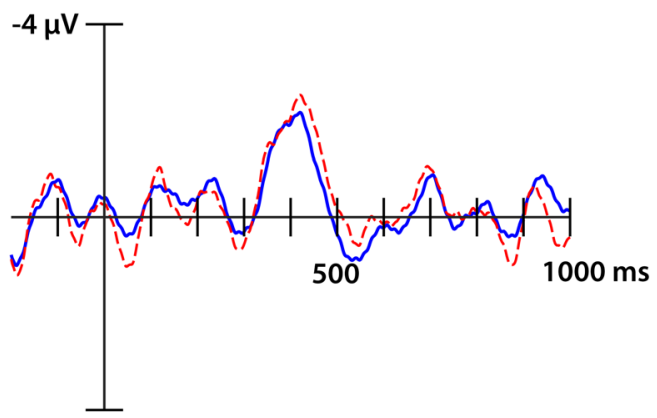

Fig. 10 Experiment 2, emotional distractor-locked, grand average waveforms at electrode site $\mathrm{Pz}$ as a function of whether the target was correctly or incorrectly identified. Panel a shows data from lag 3 trials, and panel $\mathrm{b}$ from lag 8 trials

Mean EPN amplitudes could not be estimated for six of the 156 emotional distractor words due to an inability to discern a clear component in the required time window. Mean LPP amplitudes could not be estimated for 20 emotional distractors; five due to an inability to discern a clear component in the required time window, and 15 due to lost data. The pattern of correlations between the ERP components and behavioral measures was the same as that reported in Experiment 1. Specifically, the magnitude of the EPN and LPP to the emotional distractor were each significantly positively associated with memory for the distractor and the arousal rating of the distractor, and negatively associated with target accuracy at lag 3 , but not at lag $8 .{ }^{9}$

Predicting T2 accuracy To see how much total variability in target accuracy could be explained at each lag, arousal ratings, memory performance, distractor EPN amplitude, and distractor LPP amplitude were entered as simultaneous predictors of lag 3 target accuracy in a multiple regression.

\footnotetext{
${ }^{9}$ In addition to these simple correlation analyses, further multiple regressions were conducted in order to examine the independent contributions of arousal, valence, and valence extremity to variability lag 3 target accuracy, memory, and the EPN and LPP components. In each of these multiple regressions, arousal explained significant variability even when controlling for valence and valence extremity.
} 
Results showed that, as a group, these predictors explained $32.1 \%$ of the variability in target accuracy at lag $3(\mathrm{R}=$ $0.567, F(4,125)=14.80, p<.001)$. Only memory performance (semipartial $r=-0.46, p<.001$ ) contributed significant unique variability over and above that contributed by the other predictors. When the same regression was performed on target accuracy at lag 8 , a non-significant $1.8 \%$ of the variability in target accuracy was explained $(F<1)$, and there were no significant unique predictors.

\section{Discussion}

Replicating Mathewson et al. (2008), an EIB effect was observed following taboo/sexual emotional distractor words. Also replicating Mathewson et al. (2008), performance on a surprise recognition memory test following the RSVP task was better for taboo/sexual words than for words in any of the other emotion conditions. Similar to our results in Experiment 1, and the results from Mathewson et al.'s (2008) EIB task, we also found that higher word arousal ratings predicted better memory for the emotional word and lower accuracy for subsequent targets. Additionally, the results demonstrated that memory for the word mediated the relationship between arousal and target accuracy (Fig. 8).

An enhanced EPN was detected following distractor words in the taboo/sexual condition relative to words in all other emotion conditions except the threat and anxiety conditions. Evidence for an enhanced LPP for the taboo/sexual words relative to neutral words was only found at $550 \mathrm{~ms}$ using item analysis. However, both the EPN and LPP amplitudes for emotional distractors were positively correlated with arousal ratings and memory scores, and negatively correlated with short lag target accuracy, as was found for Experiment 1. As in Experiment 1, the pattern of correlational results suggests that there was increased activation for arousing distractor words, and that this increased activation enhanced encoding into memory, and/or resulted from the encoding into memory. The results also suggest that this enhanced encoding of arousing distractor words occurred at the expense of encoding for closely trailing target items. The present results replicate those of Kennedy and colleagues (2014), who also found that negative emotional distractor images elicited enhanced N2 (EPN) and P3b (LPP) components in the EIB task. These authors also reported that the amplitude of these components following the distractor stimulus was inversely related to their amplitude following the target stimulus. Similarly, in Experiment 2 at the short lag we observed a tradeoff between the amplitude of the LPP to the distractor and the LPP to the target: the distractor LPP was larger for incorrect target trials compared to correct target trials, and EPNs and LPPs to targets were greater on target correct trials.

Interestingly, in the present experiment evidence for an enhanced positivity $700-750 \mathrm{~ms}$ after taboo/sexual words was also found. Subsequent examination of the ERP waves from Experiment 2 (Figs. 9a and 10a) suggested that this increased positivity may reflect a reduced target item EPN on short lag trials, given that the timing was correct for a targetitem EPN and the increased positivity, interpreted as reduced EPN, was present only on short-lag trials - the only lag where the target EPN would appear in that temporal window. As a result, the current ERP results also provide some evidence of reduced target processing at short lags following taboo/sexual distractor words, consistent with the results found by Kennedy and colleagues (2014).

The results from Experiment 2 provide behavioral and electrophysiological evidence for the idea that arousing words receive preferential processing when presented as to-beignored distractor items in an RSVP stream, as compared to non-arousing words.

\section{Relationships across experiments}

Mathewson et al. (2008) reported that words that were more likely to be recognized in the $\mathrm{AB}$ task were also more likely to be recognized in the capture task $(r=.80)$. Also, emotion words that led to $\mathrm{T} 2$ report failure in the $\mathrm{AB}$ task were roughly the same emotion words that led to target report failure in the capture task $(r=.50)$. However, in Mathewson et al. the same participants performed both paradigms in a single session. In the present work, different participants performed the $\mathrm{AB}$ task in Experiment 1 and the EIB task in Experiment 2. Regardless, we observed the same pattern as Mathewson et al. where words that were more likely to be recognized in the $\mathrm{AB}$ task were also more likely to be recognized in the capture task $(r=$ $.81, p<.001$ ), and words that led to $\mathrm{T} 2$ report failure in the $\mathrm{AB}$ task were roughly the same emotion words that led to target report failure in the capture task $(r=.45, p<.001)$.

\section{General discussion}

The main purpose of the current study was to use electrophysiological recording (ERPs) to examine the mechanisms underlying the $\mathrm{AB}$ with emotional words as $\mathrm{T} 1$, and $\mathrm{EIB}$ effects with emotional word distractors. We hypothesized that the $\mathrm{AB}$ and EIB effects, which have typically been reported in distinct studies, shared common underlying neurocognitive mechanisms, at least in part, and would thus show comparable patterns of behavioral and ERP results when investigated using the same stimuli. We conducted two experiments, to compare the impact of emotionally arousing words in dualtask (AB) and single-task (EIB) RSVP paradigms.

Replicating the work of Mathewson and colleagues (2008), the behavioral results demonstrated that emotionally arousing words both increased the magnitude of the $\mathrm{AB}$ when presented in the $\mathrm{T} 1$ position of an $\mathrm{AB}$ task, and set off an EIB when 
presented as to-be-ignored distractors in single-task RSVP. Consistent with the results of Mathewson and colleagues (2008), the results from the current experiments suggest that more highly arousing words presented in RSVP are better encoded into memory, resulting in poorer performance on targets that closely follow the arousing word in both $\mathrm{AB}$ and EIB tasks (Figs. 3 and 8).

It was expected that emotionally arousing $\mathrm{T} 1$ words in the $\mathrm{AB}$ task used in Experiment 1 would evoke ERP effects similar to those resulting from emotionally arousing distractor words in the EIB task used in Experiment 2, given that the same emotion words were used, and given evidence that both effects are a result of encoding limitations in a limited capacity system (Kennedy et al., 2014; Kranczioch et al., 2007; Sergent et al., 2005; Shapiro et al., 2006). Specifically, given the results from Kennedy and colleagues (2014), it was expected that the amplitude of both the EPN and LPP components would be increased following emotionally arousing words, regardless of the task in which they were presented. These increases in EPN and LPP amplitude were also expected to be predictive of memory for the emotional words and poorer target accuracy at lag 3 , but not lag 8 , in both tasks. These predictions were supported, as the EPN and LPP were both significantly related to arousal scores in both $\mathrm{AB}$ and EIB tasks, and for both tasks the amplitudes of these components were positively related to memory for the emotional word and negatively related to lag 3 target accuracy, but not lag 8 accuracy.

Furthermore, consistent with the tradeoff between $\mathrm{T} 1$ and $\mathrm{T} 2$ processing in the $\mathrm{AB}$ paradigm (e.g., Kranczioch et al., 2007; Sergent et al., 2005; Shapiro et al., 2006), and the tradeoff between emotional distractor and target processing in the EIB paradigm (Kennedy et al., 2014), a tradeoff was observed here between the amplitude of the LPP to the T1/ distractor and the LPP to the T2/target. In both experiments, the T1/distractor LPP was larger for incorrect T2/target trials compared to correct trials, and the EPNs and LPPs to T2/target were greater on correct trials. This tradeoff was also observed with the correlations where greater EPN and LPP amplitudes to $\mathrm{T} 1$ predicted reduced T2 accuracy in Experiment 1, and greater EPN and LPP amplitudes to the emotional distractor predicted reduced target accuracy in Experiment 2.

\section{Early posterior negativity}

In both experiments, the EPN component was significantly enhanced approximately $400-450 \mathrm{~ms}$ following sexual/taboo T1/distractor words (shown in Figs. 5b and 9b). Furthermore, in both experiments, greater EPN amplitude was associated with more highly arousing words, better memory for associated words, and reduced accuracy for targets closely following the associated word. Previous ERP studies of the AB and EIB effects (Kennedy et al., 2014; Kranczioch et al., 2007; Sergent et al., 2005; Shapiro et al. 2006) have suggested that the EPN component indexes the early portion of the capacity-limited stage in the central interference model of the AB. More specifically, the EPN has been suggested to index a stage responsible for selecting a relevant object among distractors (Kennedy et al., 2014; Woodman et al., 2009), and has been linked to automatic allocation of visual attention to stimuli of motivational significance (Junghöfer et al., 2001; Kissler et al., 2009; Schupp et al., 2006). Therefore, the current results suggest that emotionally arousing stimuli in RSVP may trigger a response from a mechanism indexed by the EPN that selects stimuli of motivational significance for further processing in a limited-capacity system such as working memory. These results also suggest that emotionally arousing stimuli trigger a response from this mechanism regardless of whether these stimuli are the subject of an active search. This consistent EPN effect in both RSVP tasks is consistent with previous research demonstrating that the EPN response to emotional words is a robust phenomenon that occurs even in the presence of interference from another task (Kissler et al., 2009).

However, while the amplitude of the LPP to the T1/ distractor showed a tradeoff with the amplitude of the EPN and LPP to the T2/target, there was no tradeoff between the EPN to T1/distractor and the amplitude of the EPN or LPP to the T2/target in either experiment. Furthermore, the T1/ distractor EPN did not differ for correct and incorrect short lag trials. This is interesting as it suggests that although the EPN and LPP to the T1/distractor are both enhanced for the sexual words, it may be the encoding into working memory of $\mathrm{T} 1 /$ distractor that has the most direct implications for T2/ target processing and accuracy. This also matches the conclusions of the multiple regressions where LPP amplitude to T1/ critical distractor predicted T2/target accuracy over and above T1/distractor EPN amplitude, but T1/distractor EPN amplitude was not a significant predictor of $\mathrm{T} 2 /$ target accuracy after controlling for T1/distractor LPP amplitude. These results are also consistent with those of Kennedy et al. (2014) who found that although the EPN to the emotional distractor was later on incorrect trials than on correct trials, the amplitude of the EPN to the emotional distractor did not differ for correct and incorrect target trials.

\section{Late positive potential}

In Experiment 1 an enhanced LPP was found following sexual/ taboo words (520-600 ms) when they were presented as T1 items, and greater LPP amplitude was correlated with higher word arousal ratings, better memory for those words, and reduced accuracy for closely trailing T2s. There was also the predicted tradeoff between the amplitude of the LPP to the T1/distractor and the LPP to the T2/target where the T1/distractor LPP was larger for incorrect $\mathrm{T} 2 /$ target trials compared to correct trials, and LPPs to the T2/target were larger on correct trials. In Experiment 2 there was less differentiation of the LPP for emotional 
distractors, though an enhanced LPP was found following sexual/taboo distractor words at $550 \mathrm{~ms}$ in the item analysis. However, LPP amplitude in Experiment 2 was significantly related to higher word arousal ratings, better memory for those words, and reduced accuracy for closely trailing T2s as in Experiment 1. It is possible that that the arousal-induced enhancement of the LPP is more robust for words that are targets of an active search (e.g., T1 items) as compared to distractor items; however, similar patterns in LPP result are present in both cases. This suggestion is consistent with previous work that has demonstrated that the $\mathrm{P} 300$ is most prominent following stimuli that are task-relevant (e.g., Picton, 1992).

Given the notion that the P300 indexes encoding of information into working memory, it makes sense that the LPP would be larger in Experiment 1 than in Experiment 2 since only in the first experiment were participants instructed to remember these words; in Experiment 2 they were to-be-ignored distractors. In addition to being more task-relevant in Experiment 1, T1 was also likely more physically salient in Experiment 1 where it was the lone red-colored item, than the emotional distractor was in Experiment 2 where it was the same color (black) as the filler items. Indeed, examination of memory for the T1 words in Experiment 1 (Table 1) and emotional distractor words in Experiment 2 (Table 3) shows that the words were remembered by roughly half as many participants in Experiment 2. Therefore, the current results provide evidence that the LPP, like the EPN, indexes a mechanism that results in greater processing of stimuli in working memory and thereby contributes to the central interference that results in the enhancement of the $\mathrm{AB}$ effect and creation of the EIB effect. These results are consistent with previous research highlighting the positive correlation between LPP (P3/M300) amplitude and AB magnitude (Shapiro et al., 2006), and research highlighting the distractor/T1 and T2 processing tradeoff (Kennedy et al., 2014; Sergent et al., 2005).

Dell'Acqua and colleagues (Dell'Acqua, Doro, Dux, Losier, \& Jolicoeur, 2016; Dell'Acqua et al., 2015) provided evidence for a frontal $\mathrm{P} 3 \mathrm{a}$ component to $\mathrm{AB}$ targets that was dissociable from the parietal P3b component, referred to as the LPP here. These authors provided evidence that the frontal P3a reflected detection of the relevant target(s), whereas the $\mathrm{P} 3 \mathrm{~b}$ reflected encoding of the target(s). They posited that the $\mathrm{AB}$ delays $\mathrm{T} 2$ encoding into working memory, and impairs, but does not slow down detection of $\mathrm{T} 2$. In the present study we did not have a target absent condition that would have allowed us to clearly discern the P3a component, but in future studies it would be interesting to examine whether the $\mathrm{P} 3 \mathrm{a}$ differs with emotion condition in the $\mathrm{AB}$ paradigm, and whether a $\mathrm{P} 3 \mathrm{a}$ would be observed in the EIB paradigm, and if so whether this would vary with emotion condition.

\section{Do emotion induced blindness and emotion-induced enhancement of attentional blink share common mechanisms?}

The main goal of the current study was to examine whether EIB and the emotion-induced enhancement of the $\mathrm{AB}$ are a result of similar underlying mechanisms. Previous research has suggested that these phenomena may arise through shared mechanisms (Kennedy et al., 2014); however, prior to this, study no ERP research has allowed a side-by-side comparison using similar behavioral methodology and stimuli, and no study has examined ERPs to emotional T1s in an AB paradigm. Replicating previous research, the similar behavioral results from the two tasks in this study support the sharedmechanism interpretation of the EIB and emotion-enhanced AB. This shared-mechanism interpretation is further supported by the enhanced EPNs and LPPs to arousing words in both tasks - ERP enhancements that predict improved memory for the arousing words at the expense of report accuracy for closely trailing targets. Furthermore, in both tasks the LPP showed a tradeoff between T1 and T2. Prior research suggested that the EPN is associated with a stage prior to representation of a stimulus in working memory - such as selection of a relevant object among distractors (Woodman et al., 2009; Kennedy et al., 2014) - and concurrently suggested that the LPP indexes representation in working memory (Kranczioch et al., 2007; Sergent et al., 2005; Vogel et al., 1998; Vogel \& Luck, 2002). Thus, the current results provide evidence that in both $\mathrm{AB}$ and EIB paradigms arousing words receive enhanced attentional selection and enhanced encoding into working memory, and that this enhanced encoding into working memory comes at the expense of processing resources and accuracy for closely trailing emotionally-neutral targets.

Based on the current results, several answers can be provided to the question of whether the EIB and emotion-induced enhancement of the $\mathrm{AB}$ have shared underlying mechanisms. First, the phenomenological similarity between the behavioral results in these tasks is robust and has been observed in other studies (Mathewson et al., 2008). Second, the processing indexed by the EPN - thought to represent a semantic classification mechanism (Schupp et al., 2006; Kissler et al., 2009) that results in the selection of a stimulus among distractors for further processing in working memory (Woodman et al., 2009; Kennedy et al., 2014) - is clearly enhanced for sexual words in both the EIB and AB paradigms. The robust EPN following emotional words, regardless of whether the word was a target item or distractor, is consistent with previous research demonstrating that the EPN response to emotional words is a robust phenomenon that occurs even in the presence of interference (Kissler et al., 2009). However, in both experiments the T1/distractor EPN did not differ for short lag correct and incorrect trials, suggesting that although the EPN and LPP to the T1/distractor are both enhanced for sexual words, it is the encoding into working memory of $\mathrm{T} 1 /$ distractor that has 
the most direct implications for T2/target accuracy. Third, the presence of a LPP in both the AB and EIB experiments, its significant correlations with behavioral performance in both tasks, and the tradeoff in LPP magnitude for T1/emotional distractors and T2/targets - is consistent with the idea that the LPP is associated with encoding items into working memory. Along with the results of Kennedy and colleagues (2014), the present results suggest that salient emotional items may garner enhanced LPPs both due to their task relevance and to their arousing nature. Furthermore, the larger LPP that was observed in Experiment 1 , when the arousing words were task-relevant, suggests that these routes may be somewhat additive in nature. Overall, our results suggest that $\mathrm{EIB}$ and emotion-enhanced $\mathrm{AB}$ arise through similar underlying mechanisms that are involved in attentional selection and working memory encoding and can be observed in EPN and LPP components.

However, it is worth noting that despite the similarities observed here, dissociations have been observed for the two paradigms. For example, while the $\mathrm{AB}$ can observed when $\mathrm{T} 1$ and $\mathrm{T} 2$ appear in different spatial locations (e.g., Duncan, Ward, \& Shapiro, 1994; Visser, Zuvic, Bischof, \& DiLollo, 1999), Most and Wang (2011) provided evidence that EIB was only observed for targets that appeared in the same spatial location as the emotional distractor. It is also unclear whether lag-1 sparing - a sparing of the target deficit at lag 1 where the target comes immediately after T1/emotional distractor - can be found with the EIB paradigm (Kennedy \& Most, 2015), whereas lag-1 sparing is found fairly often in the AB paradigm (see Hommel \& Akyürek, 2005; Visser, Bischof, \& Di Lollo, 1999). Also, in the EIB paradigm Kennedy et al. (2014) observed a distractor-related late positivity that was enhanced for negative distractors relative to neutral distractors and was larger on target incorrect trials. They suggested that this may be a $\mathrm{P}_{\mathrm{D}}$ component reflecting the extra difficulty in disengaging attention from emotional distractors. If, so we may not expect this to be observed in the $\mathrm{AB}$ paradigm with emotional $\mathrm{T} 1 \mathrm{~s}$ that are task relevant, but this late positivity was not examined here for either paradigm. Also, as noted above, the P3a component observed by Dell'Acqua and colleagues in the $\mathrm{AB}$ paradigm (Dell'Acqua et al., 2015, 2016) was also not examined here as a function of emotion condition for either the $\mathrm{AB}$ or EIB paradigms. Therefore, at this point, we suggest caution in concluding that EIB and the emotional AB share completely overlapping mechanisms. At present, it appears safest to conclude that $\mathrm{EIB}$ and the emotional $\mathrm{AB}$ are related, and share similar underlying mechanisms, at least in part.

Author note This research was supported by the Natural Sciences and Engineering Research Council of Canada (NSERC), the Canada Foundation for Innovation (CFI), and the Ontario Innovation Trust (OIT).

\section{References}

Anderson, A. K. (2005). Affective influences on the attentional dynamics supporting awareness. Journal of Experimental Psychology General, 134(2), 258-281. doi:10.1037/0096-3445.134.2.258

Aquino, J. M., \& Arnell, K. M. (2007). Attention and the processing of emotional words: Dissociating effects of arousal. Psychonomic Bulletin and Review, 14, 430-435.

Arnell, K. M., Killman, K. V., \& Fijavz, D. (2007). Blinded by emotion: Target misses follow attention capture by arousing distractors in RSVP. Emotion, 7(3), 465-477. doi:10.1037/1528-3542.7.3.465

Baron, R. M., \& Kenny, D. A. (1986). The moderator-mediator variable distinction in social psychological research: Conceptual, strategic, and statistical considerations. Journal of Personality and Social Psychology, 51(6), 1173-1182. doi:10.1037//0022-3514.51.6.1173

Batterink, L., Karns, C. M., Yamada, Y., \& Neville, H. (2010). The role of awareness in semantic and syntactic processing: An ERP attentional blink study. Journal of Cognitive Neuroscience, 22(11), 2514-2529. doi:10.1162/jocn.2009.21361

Chun, M. M., \& Potter, M. C. (1995). A two-stage model for multiple target detection in rapid serial visual presentation. Journal of Experimental Psychology: Human Perception and Performance, 21(1), 109-127. doi:10.1037//0096-1523.21.1.109

Dell'Acqua, R., Doro, M., Dux, P. E., Losier, T., \& Jolicœur, P. (2016). Enhanced frontal activation underlies sparing from the attentional blink: Evidence from human electrophysiology. Psychophysiology, 53, 623-633.

Dell'Acqua, R., Dux, P. E., Wyble, B., Doro, M., Sessa, P., Meconi, F., \& Jolicœur, P. (2015). The attentional blink impairs detection and delays encoding of visual information: Evidence from human electrophysiology. Journal of Cognitive Neuroscience, 27(4), 720-735.

Donchin, E. (1981). Surprise!... surprise? Psychophysiology, 18(5), 493513. doi:10.1111/j.1469-8986.1981.tb01815.x

Duncan, J., Ward, R., \& Shapiro, K. (1994). Direct measurement of attentional dwell time in human vision. Nature, 369(6478), 313-315.

Frischen, A., Eastwood, J. D., \& Smilek, D. (2008). Visual search for faces with emotional expressions. Psychological Bulletin, 134(5), 662-676. doi:10.1037/0033-2909.134.5.662

Hommel, B., \& Akyürek, E. G. (2005). Lag-1 sparing in the attentional blink: Benefits and costs of integrating two events into a single episode. The Quarterly Journal of Experimental Psychology Section A, 58(8), 1415-1433.

Jolicoeur, P. (1999). Concurrent response-selection demands modulate the attentional blink. Journal of Experimental Psychology: Human Perception and Performance, 25(4), 1097-1113. doi:10.1037// 0096-1523.25.4.1097

Jolicoeur, P., \& Dell'Acqua, R. (1998). The demonstration of short-term consolidation. Cognitive Psychology, 36(2), 138-202. doi:10.1006/ cogp.1998.0684

Junghöfer, M., Bradley, M. M., Elbert, T. R., \& Lang, P. J. (2001). Fleeting images: A new look at early emotion discrimination. Psychophysiology, 38(2), 175-178. Retrieved from http:/www. ncbi.nlm.nih.gov/pubmed/11347862

Keil, A., \& Ihssen, N. (2004). Identification facilitation for emotionally arousing verbs during the attentional blink. Emotion, 4(1), 23. doi: 10.1037/1528-3542.4.1.23

Kennedy, B. L., Most, S. B. (2015). The rapid perceptual impact of emotional distractors. PLoS ONE 10(6): e0129320. doi:10.1371/ journal.pone. 0129320

Kennedy, B. L., Rawding, J., Most, S. B., \& Hoffman, J. E. (2014). Emotioninduced blindness reflects competition at early and late processing stages: An ERP study. Cognitive, Affective, \& Behavioral Neuroscience, 14(4), 1485-1498. doi:10.3758/s13415-014-0303-x 
Kissler, J., Herbert, C., Winkler, I., \& Junghofer, M. (2009). Emotion and attention in visual word processing: An ERP study. Biological Psychology, 80(1), 75-83. doi:10.1016/j.biopsycho.2008.03.004

Kranczioch, C., Debener, S., Maye, A., \& Engel, A. K. (2007). Temporal dynamics of access to consciousness in the attentional blink. NeuroImage, 37(3), 947-955. doi:10.1016/j.neuroimage.2007.05.044

Luck, S. J., Vogel, E. K., \& Shapiro, K. L. (1996). Word meanings can be accessed but not reported during the attentional blink. Nature, 383(6601), 616-618.

MacKay, D. G., Shafto, M., Taylor, J. K., Marian, D. E., Abrams, L., \& Dyer, J. R. (2004). Relations between emotion, memory, and attention: Evidence from taboo Stroop, lexical decision, and immediate memory tasks. Memory \& Cognition, 32(3), 474-488. doi:10.3758/ BF03195840.

Mathewson, K. J., Arnell, K. M., \& Mansfield, C. A. (2008). Capturing and holding attention: The impact of emotional words in rapid serial visual presentation. Memory \& Cognition, 36(1), 182-200. doi:10. 3758/MC.36.1.182

McHugo, M., Olatunji, B. O., \& Zald, D. H. (2013). The emotional attentional blink: What we know so far. Frontiers in Human Neuroscience, 7. doi:10.3389/fnhum.2013.00151

McKenna, F., \& Sharma, D. (1995). Intrusive cognitions: An investigation of the emotional Stroop task. Journal of Experimental Psychology: Learning, Memory, and Cognition, 21(6), 15951607. doi:10.1037//0278-7393.21.6.1595

Milders, M., Sahraie, A., Logan, S., \& Donnellon, N. (2006). Awareness of faces is modulated by their emotional meaning. Emotion, 6(1), 10 . doi:10.1037/1528-3542.6.1.10

Most, S. B., Chun, M. M., Widders, D. M., \& Zald, D. H. (2005). Attentional rubbernecking: Cognitive control and personality in emotion-induced blindness. Psychonomic Bulletin \& Review, 12(4), 654-661. Retrieved from http://www.ncbi.nlm.nih.gov/ pubmed/16447378

Most, S. B., Smith, S. D., Cooter, A. B., Levy, B. N., \& Zald, D. H. (2007). The naked truth: Positive, arousing distractors impair rapid target perception. Cognition \& Emotion, 21(5), 964-981. doi:10. 1080/02699930600959340

Most, S. B., \& Wang, L. (2011). Dissociating spatial attention and awareness in emotion-induced blindness. Psychological Science, 22(3), 300-305.

Picton, T. W. (1992). The P300 wave of the human event-related potential. Journal of Clinical Neurophysiology, 9(4), 456-479.

Potter, M. C. (1993). Very short-term conceptual memory. Memory \& Cognition, 21(2), 156-161.

Potter, M. C., \& Lombardi, L. (1990). Regeneration in the short-term recall of sentences. Journal of Memory and Language, 29(6), $633-654$.

Raymond, J. E., Shapiro, K. L., \& Arnell, K. M. (1992). Temporary suppression of visual processing in an RSVP task: An attentional blink? Journal of Experimental Psychology: Human Perception and Performance, 18(3), 849-860. doi:10.1037//0096-1523.18.3.849

Schacht, A., \& Sommer, W. (2009a). Emotions in word and face processing: Early and late cortical responses. Brain and Cognition, 69(3), 538-550. doi:10.1016/j.bandc.2008.11.005

Schacht, A., \& Sommer, W. (2009b). Time course and task dependence of emotion effects in word processing. Cognitive, Affective, \& Behavioral Neuroscience, 9(1), 28-43. doi:10.3758/CABN.9.1.28

Schupp, H. T., Cuthbert, B. N., Bradley, M. M., Cacioppo, J. T., Ito, T., \& Lang, P. J. (2000). Affective picture processing: The late positive potential is modulated by motivational relevance. Psychophysiology, 37(2), 257-261. doi:10.1111/1469-8986. 3720257

Schupp, H., Cuthbert, B., Bradley, M., Hillman, C., Hamm, A., \& Lang, P. (2004). Brain processes in emotional perception: Motivated attention. Cognition \& Emotion, 18(5), 593-611. doi:10.1080/ 02699930341000239

Schupp, H. T., Flaisch, T., Stockburger, J., \& Junghöfer, M. (2006). Emotion and attention: Event-related brain potential studies. Progress in Brain Research, 156, 31-51. doi:10.1016/S00796123(06)56002-9

Schupp, H. T., Junghöfer, M., Weike, A. I., \& Hamm, A. O. (2003a). Attention and emotion: An ERP analysis of facilitated emotional stimulus processing. Neuroreport, 14(8), 1107-1110. doi:10.1097/ 01.wnr.0000075416.59944.49

Schupp, H., Junghöfer, M., Weike, A. I., \& Hamm, A. O. (2003b). Emotional facilitation of sensory processing in the visual cortex. Psychological Science, 14(1), 7-13. Retrieved from http://pss. sagepub.com/content/14/1/7.short

Schupp, H. T., Junghöfer, M., Weike, A. I., \& Hamm, A. O. (2004). The selective processing of briefly presented affective pictures: An ERP analysis. Psychophysiology, 41(3), 441-449. doi:10.1111/j.14698986.2004.00174

Schupp, H. T., Ohman, A., Junghöfer, M., Weike, A. I., Stockburger, J., \& Hamm, A. O. (2004). The facilitated processing of threatening faces: An ERP analysis. Emotion, 4(2), 189-200. doi:10.1037/1528-3542. 4.2.189

Schupp, H. T., Stockburger, J., Codispoti, M., Junghöfer, M., Weike, A. I., \& Hamm, A. O. (2007). Selective visual attention to emotion. The Journal of Neuroscience: The Official Journal of the Society for Neuroscience, 27(5), 1082-1089. doi:10.1523/JNEUROSCI.322306.2007

Schwabe, L., Merz, C. J., Walter, B., Vaitl, D., Wolf, O. T., \& Stark, R. (2011). Emotional modulation of the attentional blink: The neural structures involved in capturing and holding attention. Neuropsychologia, 49(3), 416-425.

Schwabe, L., \& Wolf, O. T. (2010). Emotional modulation of the attentional blink: Is there an effect of stress? Emotion, 10(2), 283. doi:10. 1037/a0017751

Sergent, C., Baillet, S., \& Dehaene, S. (2005). Timing of the brain events underlying access to consciousness during the attentional blink. Nature Neuroscience, 8(10), 1391-1400. doi:10.1038/nn1549

Shapiro, K., Schmitz, F., Martens, S., Hommel, B., \& Schnitzler, A. (2006). Resource sharing in the attentional blink. NeuroReport, 17(2), 163-166. doi:10.1097/01.wnr.0000195670.37892.1a

Visser, T. A., Bischof, W. F., \& Di Lollo, V. (1999). Attentional switching in spatial and nonspatial domains: Evidence from the attentional blink. Psychological Bulletin, 125(4), 458.

Visser, T. A., Zuvic, S. M., Bischof, W. F., \& Di Lollo, V. (1999). The attentional blink with targets in different spatial locations. Psychonomic Bulletin \& Review, 6(3), 432-436.

Vogel, E. K., \& Luck, S. J. (2002). Delayed working memory consolidation during the attentional blink. Psychonomic Bulletin \& Review, 9(4), 739-743. doi:10.3758/BF03196329

Vogel, E. K., Luck, S. J., \& Shapiro, K. L. (1998). Electrophysiological evidence for a postperceptual locus of suppression during the attentional blink. Journal of Experimental Psychology Human Perception and Performance, 24(6), 1656-1674. Retrieved from http://www.ncbi.nlm.nih.gov/pubmed/9861716

Vogt, J., De Houwer, J., Koster, E. H. W., Van Damme, S., \& Crombez, G. (2008). Allocation of spatial attention to emotional stimuli depends upon arousal and not valence. Emotion, 8(6), 880-885. doi:10.1037/ a0013981

Woodman, G. F., Arita, J. T., \& Luck, S. J. (2009). A cuing study of the N2pc component: An index of attentional deployment to objects rather than spatial locations. Brain Research, 1297, 101-111. doi: 10.1016/j.brainres.2009.08.011 